\title{
Applying Registry Services to Spaceflight Technologies to Aid in the Assignment of Assigned Numbers to Disparate Systems and their Technologies to further enable Interoperability
}

\author{
Robert N. Bradford ${ }^{1}$ and Kelvin F. Nichols ${ }^{2}$ \\ NASA/Marshall Space Flight Center, MSFC, Alabama, 35812 \\ Keith R. Witherspoon ${ }^{3}$ \\ Universities Space Research Association, Huntsville, Alabama, 35806
}

\section{Abstract}

To date very little effort has been made to provide interoperability between various space agency projects. To effectively get to the Moon and beyond systems must interoperate. To provide interoperability, standardization and registries of various technologies will be required. These registries will be created as they relate to space flight. With the new NASA Moon/Mars initiative, a requirement to standardize and control the naming conventions of very disparate systems and technologies is emerging. The need to provide numbering to the many processes, schemas, vehicles, robots, space suits and technologies (e.g. versions), to name a few, in the highly complex Constellation initiative is imperative. The number of corporations, developer personnel, system interfaces, people interfaces will require standardization and registries on a scale not currently envisioned. It would only take one exception (stove piped system development) to weaken, if not, destroy interoperability.

To start, a standardized registry process must be defined that allows many differing engineers, organizations and operators the ability to easily access disparate registry information across numerous technological and scientific disciplines. Once registries are standardized the need to provide registry support in terms of setup and operations, resolution of conflicts between registries and other issues will need to be addressed. Registries should not be confused with repositories. No end user data is "stored" in a registry nor is it a configuration control system.

Once a registry standard is created and approved, the technologies that should be registered must be identified and prioritized. In this paper, we will identify and define a registry process that is compatible with the Constellation initiative and other non related space activities and organizations. We will then identify and define the various technologies that should use a registry to provide interoperability. The first set of technologies will be those that are currently in need of expansion namely the assignment of satellite designations and the process which controls assignments. Second, we will analyze the technologies currently standardized under the Consultative Committee for Space Data Systems (CCSDS) banner. Third, we will analyze the current CCSDS working group and Birds of a Feather $(\mathrm{BoF})$ activities to ascertain registry requirements. Lastly, we will identify technologies that are either currently under the auspices of another standards body or technologies that are currently not standardized. For activities one through three, we will provide the analysis by either discipline or technology with rationale, identification and brief description of requirements and precedence. For activity four, we will provide a list of current standards bodies e.g. IETF and a list of potential candidates.

\section{Introduction}

$\mathrm{T}$ he scope and dynamics of space operations are changing. With the announcement that the USA will return to the Moon and eventually to Mars, a new dynamics in space systems and operations is emerging. New information based technologies are evolving that will change how we use Information Technology (IT) in future space operations. As the Internet evolved, it became clear that registries like the Internet Assigned Number Authority (IANA) were necessary to control and provide access to those users, developers and others, who were developing the technologies and the users who needed the information that traveled on it. What has made the Internet functional is the fact that it is interoperable. All anyone has to do is plug his device into a network and, regardless of the functionality of the device; it can communicate to virtually anywhere. The only way to accomplish this type of interoperability and control was to establish the IANA registry. This approach over time has proven to

\footnotetext{
${ }^{1}$ Manager, Data Systems, Mission Operations Laboratory, Mail Code: EO40.

${ }^{2}$ Engineer, Mission Operations Laboratory, Mail Code: EO40.

${ }^{3}$ Student Intern, University Space Research Association, Huntsville, AL
} 
be effective. Now as we contemplate what will be required to return to the Moon and on to Mars, questions arise concerning current and future technologies that will be required to enable interoperability and what mechanisms will be necessary to control them. We know from past experience, that there will be a significant requirement to insure unique numbering of space vehicles, satellites, robots and bases. We, the space flight community, must identify and implement interoperability and control policies and processes including access methodologies. These must be conducive to developer, manager and the general user populations. This conduciveness must be in terms of process, ease of access and understanding (lack of complexity). If interoperability is to succeed, it must be understood and implemented at all levels. From the highest managers (for policy) to procurement/acquisition personnel (to ensure what is bought is interoperable), to the engineers and technicians (who will design and install) and finally to the operational personnel (who will operate and maintain this system of systems end to end). To accomplish this interoperability some sort of registry process will be required. The scope of registry services described in this paper is generally applied to the technologies used in space-based development, systems (terrestrial and extraterrestrial) and operations. Technologies that are under an established registry e.g. IANA for the Internet, that may have application to space systems will be included.

However, first within the space operations community there must be a single point for defining and standardizing registries. The definitions of the registries, how to organize and operate them, and how they interrelate must be focused within one recognized authority. Multiple registries of the same technologies would not enable interoperability but would discourage it, making unique numbering difficult if not impossible. Imagine the chaos of employing two independent registries for assigning spacecraft IDs that use the same or integrated transport mechanisms!

In this paper we will recommend an approach to resolve this situation. We will list and briefly describe; the technologies currently being used publicly and within the space community that are already supported by a registry e.g. the Spacecraft Identifier, the CCSDS operational standards to ascertain applicability to registries, within the current CCSDS working groups potential areas where registry services could be applied. We will look at publicly available technologies that may be applied to future space based systems not currently used in space systems.

\section{Interoperability}

A simple definition for interoperability from a dictionary.com website states that interoperability is the ability of software and hardware on multiple machines from multiple vendors to communicate. Taking the definition a little further and putting it in terms of the current problem, one can think of interoperability as the ability of having multiple systems of different types to exchange and use resources and information with little or no prior knowledge of each other's systems. Interoperability problems might not be a big concern in a small environment with a single control center or remote investigator site, but as future space operations grow and span across many different elements, it is easy to see how quickly interoperability could become an issue. One way to attack this problem is to require each control center to be designed exactly the same way. This would insure interoperability between the control centers but it may be a hard sell to the overall user community that may have a different design concept for their control center. Aside from hurting innovation, this may also be viewed as a hostile form of network dictatorship. A better approach to this problem would involve registering the control center data interface. This would allow anyone in the user community to search the registry to see how the control center data interface is defined. Now the remote user has the freedom to design his or her control center however they want without having to know the full design behind the original control center. Registries would play a key role in helping define the interfaces between these elements and could eventually take the place of Interface Control Documents (ICD) that are used to define how an element will need to design its interface to be able to communicate between elements. This would eliminate the need for multiple documents with each center, international entity, and remote investigator or developer community member.

\section{Define the Problem}

Currently there are no standards or processes to manage and control spaceflight related technologies. Granted there are many registries and standards organizations that exist to address specific areas but these areas are not related to spaceflight. To enable interoperability one aspect for success will be the ability to access specific standards and controls during design, implementation and operations.

Unique numbering will be required in all facets of space based operations ranging from frame identification associated with specific spacecraft, proximity communications between spacecraft and between ground elements and spacecraft. These unique number requirements will range from spacecraft identification, applications, processes 
to individual information schemas e.g. XML. Within the space operations community since its inception, each project or program generally developed all the necessary systems specifically for their use (fulfilling their specific requirements) without recognizing that interoperability between disparate systems and operations would be necessary. Until the Space Station was implemented this was not a serious concern especially when using expendable vehicles. Today with shrinking budgets, it is beneficial to reuse hardware and software, to be able to develop and interact with other systems and applications without significant knowledge of those systems and applications, and do so in very short time frames e.g. minutes, hours or days.

Specifically, how registries could help with future space operations is in the management of Space Craft Identifiers (SCID). A SCID is an eight to ten bit binary field that is tied to the link layer protocol and helps identify which telemetry stream is associated with a particular spacecraft or spacecraft subsystem. There will be an increasing amount of hardware and vehicles being flown in support of manned and unmanned missions to explore our solar system. A redesigned SCID registry could take control of this assignment authority and help manage the need to assign these SCID and eliminate the need to reclaim them for reuse after a mission is over. There could be safety and operational issues associated with reuse of previously assigned numbers, if numbering of specific schemas, applications and processes is involved.

Specifically, unique number of space vehicles, rovers, satellites, robots, space suits will be required well beyond what can be numbered under the present numbering scheme. As Table 1 indicates under the internationally recognized CCSDS the number of SCIDs ranges from 256 to 1024 numbers with approximately one half of these assigned to existing spacecraft. This leaves approximately 500 unique numbers unassigned. While the process allows reassignment of numbers after a program has ended, it is estimated by the authors that only 250 or so numbers are available for assignment to any major effort to return to the Moon and on to Mars. There are estimates that more than 60,000 unique assignments will be required for spacecraft IDs to go to the Moon and on to Mars. These assignments may be required for transport over RF systems, for assigning unique application numbers and for assigning and registering XML schemas.

Also some technologies have a large scale impact as far as implementation is concerned. New technologies are continually being introduced and current technologies are continually evolving. Without a registry to help keep track of these evolving technologies, developers could be wasting a lot of time reinventing solutions to problems that other developers have already solved. This situation cannot continue into the future because going back to the Moon and then on to Mars will require significant interoperability at the hardware, software, service and operational levels. Currently with some minor exceptions there are no specifications, mechanisms, processes or guidelines in place that can enable this type of interoperability. A few examples of technologies that could benefit from a registry will be covered in a different section of this paper.

\begin{tabular}{|l|l|l|l|l|}
\hline \multicolumn{1}{|c|}{ Address } & \multicolumn{1}{|c|}{$\begin{array}{l}\text { TM Space } \\
\text { Data Link } \\
\text { Protocol }\end{array}$} & $\begin{array}{l}\text { TC Space } \\
\text { Data Link } \\
\text { Protocol }\end{array}$ & $\begin{array}{l}\text { AOs Space } \\
\text { Data Link } \\
\text { Protocol }\end{array}$ & $\begin{array}{l}\text { Proximity-1 } \\
\text { Space Link } \\
\text { Protocol }\end{array}$ \\
\hline $\begin{array}{l}\text { Transfer Frame } \\
\text { Version Number } \\
\text { (TFVN) }\end{array}$ & $\begin{array}{l}\text { Always 1 } \\
\text { (binary } \\
\text { encoded } \\
\text { number is 00) }\end{array}$ & $\begin{array}{l}\text { Always 1 } \\
\text { (binary } \\
\text { encoded } \\
\text { number is 00) }\end{array}$ & $\begin{array}{l}\text { Always 2 } \\
\text { (binary } \\
\text { encoded } \\
\text { number is 01) }\end{array}$ & $\begin{array}{l}\text { Always 3 } \\
\text { (binary } \\
\text { encoded } \\
\text { number is 10) }\end{array}$ \\
\hline $\begin{array}{l}\text { Spacecraft } \\
\text { Identifier (SCID) }\end{array}$ & 0 to 1023 & 0 to 1023 & 0 to 255 & 0 to 1023 \\
\hline $\begin{array}{l}\text { Virtual Channel } \\
\text { Identifier (NCID) }\end{array}$ & 0 to 7 & 0 to 63 & 0 to 63 & 0,1 \\
\hline $\begin{array}{l}\text { Multiplexer } \\
\text { Access Point } \\
\text { identifier (MAP } \\
\text { ID) }\end{array}$ & N/A & 0 to 63 & N/A & N/A \\
\hline Port Identifier & N/A & N/A & N/A & 0 to 7 \\
\hline
\end{tabular}

Table 1, CCSDS Space Link Protocol Unique Identifiers by Protocol ${ }^{4}$ 


\section{The Solution}

The CCSDS is an international organization made up of most space agencies world wide. The CCSDS is mandated to provide standards under the International Organization for Standardization. Figure 2 shows the CCSDS organization chart, and the list of member and observers agencies and organizations is in Appendix A. Under the CCSDS charter, the Space Assigned Numbers Authority (SANA) is to provide unique numbering to the space operations community. Figure 1 provides a breakdown of the SANA. Within CCSDS it states in section 4.1.3.6 Space Assigned Numbers Authority (SANA) "The core registrar for the CMC's activities is the SANA. Many space mission protocols require that someone keep track of key protocol numbering assignments that were added after the protocol came out. Typical examples of the kinds of registries needed are for SCIDs, protocol version numbers, reserved Application Process Identifiers, and Standard Formatted Data Units (SFDU) Control Authorities. The SANA provides this key configuration management service for CCSDS. The CCSDS Management Council (CMC) approves the organization that will act as the SANA. Its public interface is focused through Web-based services provided by the Secretariat."

$\begin{array}{lll}\text { Space } & =\begin{array}{l}\text { Space flight activities (flight } \\ \text { and ground) }\end{array} \\ \text { Assigned }= & \text { Registry(ies) } \\ \text { Numbers }= & \text { Numbering scheme(s) } \\ \text { Authority }= & \begin{array}{l}\text { Controlling processes and } \\ \text { organizations }\end{array}\end{array}$

Figure 1, the Space Assigned Numbers Authority Definition Overview

The "Space" in SANA indicates the scope of the SANA which includes spaceflight activities both on the ground and in flight. There are many types of spacecraft and their integrated technologies that could possibly be provided unique numbers. The scope of SANA is all spaceflight activities that cross international boundaries. Included would be existing public registries that are applicable to spaceflight that could be approached to assign/manage

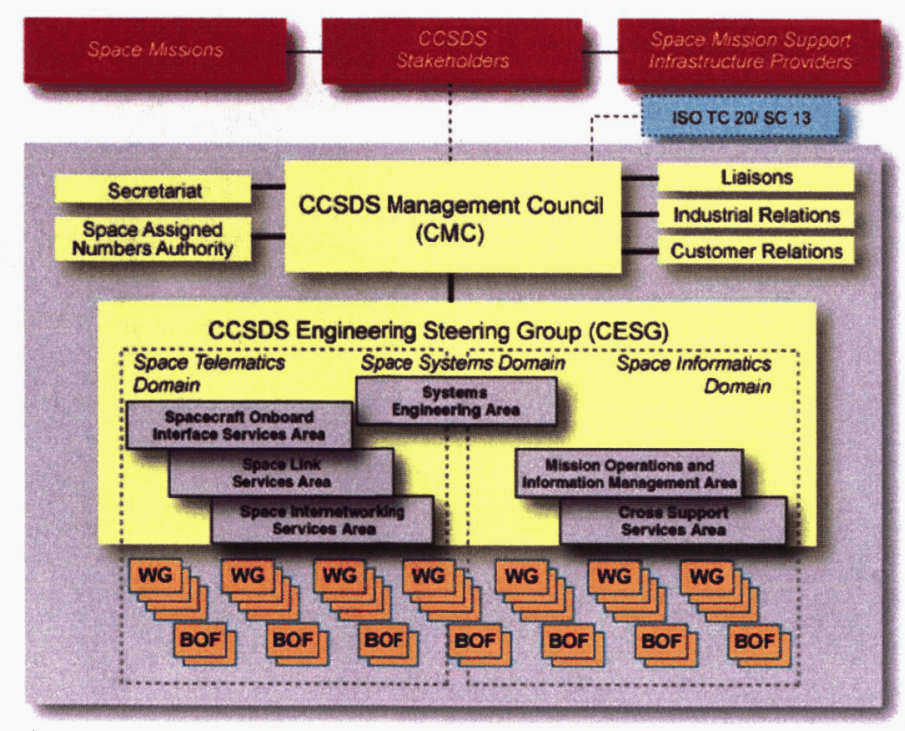

Figure 2, CCSDS Organizational Structure ${ }^{5}$ 
unique numbering of spaceflight activities under their established purview. The SANA would provide the standards and mechanisms to identify and standardize registries that would assign unique numbering to various aspects of spaceflight activities including but not limited to Earth based centers and remote terrestrial locations, vehicles, space objects, robots, satellites and bases (manned and unmanned, small and large) and potentially systems, applications and processes. The SANA should addresses public registries that support spaceflight e.g. IANA. A registry process may register ownership of hardware, formats, numbers software applications, XML schemas, especially where they cross system, program and/or national boundaries.

The "Assigned" in SANA requires a mechanism to provide numbering and infers a registry process of some sort. Numbering can be assigned by a registering process in a hierarchical registry of registries that assigns standardized control and process to the registry processes and its contents. It could assign and recognize ownership to the subregistry process and identify ownership and control that can be publicized. A registry process could provide the registration process for numbers associated with very general or very detailed level of specifics, objective associations, e.g. spacecraft (stuff you can kick) and subjective type associations, e.g. XML schemas (stuff you cannot kick).

The "Numbers" in SANA, refers to a standardized numbering scheme for all unique identification numbering approaches. The SANA needs to address various spaceflight activities that require numbering, operational situations that affect numbering, e.g. when two registered independent objects are mated, and other situations like age, use, conflicts and efficiencies e.g. number abbreviations, standards for formats, and schemas. The SANA must accomplish all of this while avoiding conflicts in numbering schemes throughout the space community.

Finally, the "Authority" in SANA, is who is in control and by what authorization for each registry. A Registry of Registries (RoR) is required to accomplish this implementation that will provide a single point for registry standardization and control. Figure 3 depicts how a RoR and the sub-registries would be related. A standard for the RoR and sub-registries would be established and the standards would apply to all sub-registries as guidelines. Also, individual sub-registry control authority would be resident in the "registered owner" of the sub-registry. The control authority for the RoR would be the CCSDS, and for sub-registries the individual agencies, programs/projects under which the sub-registry is registered. Sub-registries may be as simple as a pointer to another registry and a standardized control authority (CA) much like the CA in place today, but adjusted for the anticipated increased volume and technical diversity. Where necessary, an adjustment to the CA guidelines for specific sub-registries may be required.

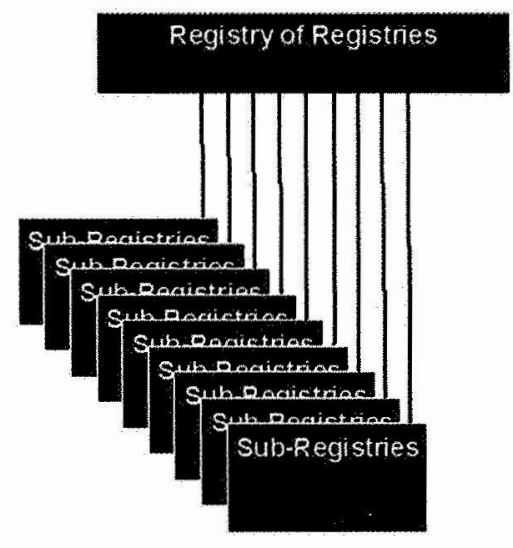

Figure 3, Registry of Registries layout

The RoR is a concept of a place to define ownership, control and access. Access to registry information is critical for interoperability where access by implementers, operators and managers is crucial. Therefore it must be able to conduct indexing of many registries and potentially pointing to the repositories associated with those registries. Under a RoR a centralized control point would be established for multiple participants to access and locate information and resources, which will provide dynamic resource integration. Having a registry of registries is necessary and essential to accomplish this. A goal is to have an entry portal into a registry of registries, which will be the single, authoritative access point to all other registries. The registry of registries would allow for a conductive 
search across the whole scope of registries. Figure 4 shows the registry of registries layout depicting a split between space organizations and public sector registry organizations. The top block in the RoR would act as the portal into the registry of registries and the sub registries below. Below the RoR, embedded in sub-registries, would be registries for any number of registered technologies such as the SCID, interface control definitions and XML schemas.

As Figure 5 depicts, Spacecraft IDs and its control authority would be controlled as it is today by CCSDS, but through a newly defined approach and process. Potentially a new approach to numbering based on future requirements should be developed. Through the CCSDS standards development process new numbering and control authority approaches and procedures could be developed.

Between the RoR and the sub-registries, a process for coordination, control and problem resolution will be required as depicted in Figure 6. There will undoubtedly be coordination needed between the RoR and subregistries in instances of defining scopes, procedures and contacts. Control issues will be resolved through this process. Any problems arising during implementation and operations will need to be resolved. As shown, two existing registry requirements are $\mathrm{XML}$ schemas and asynchronous messaging.

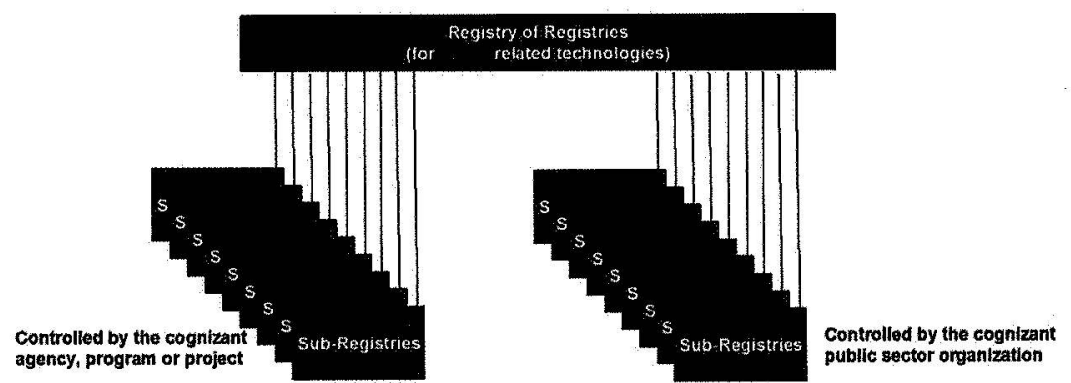

Figure 4, Registry of Registries Breakdown by Space Organizations and the Public Sector

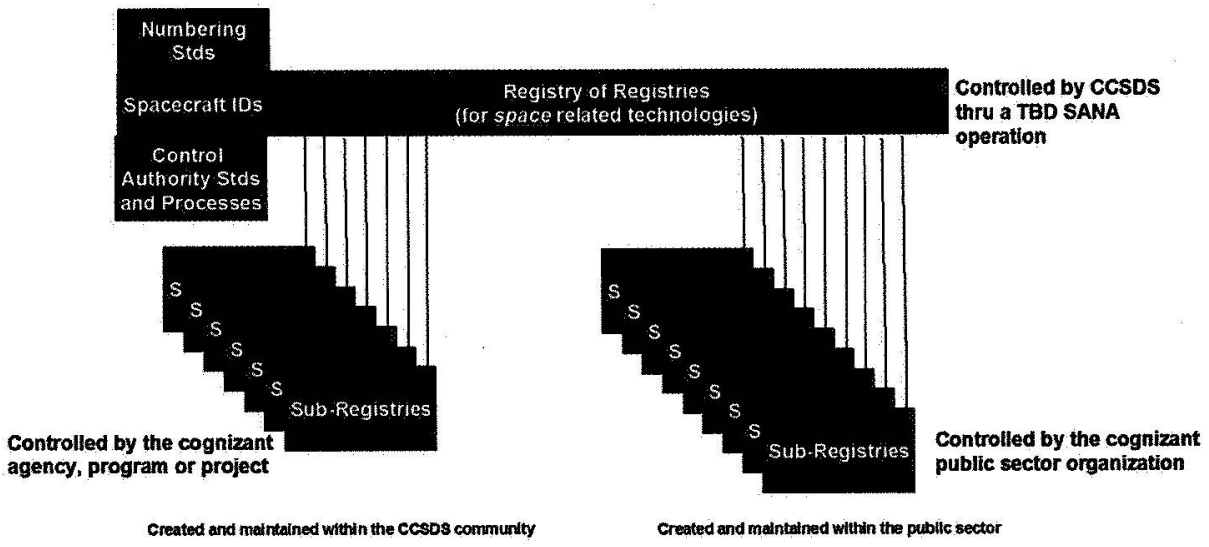

Figure 5, Registry of Registries Spacecraft IDs and Control Authority 

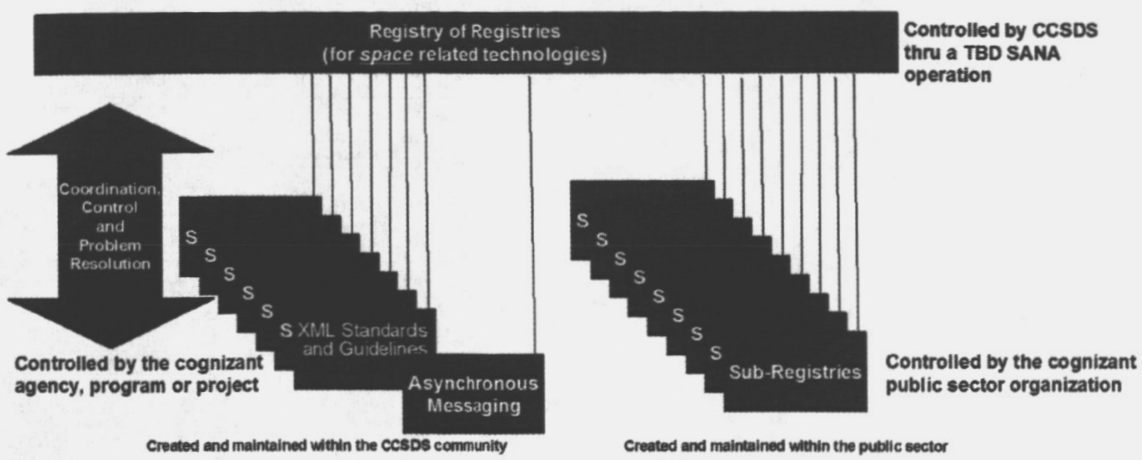

Figure 6, Registry of Registries Process and Examples

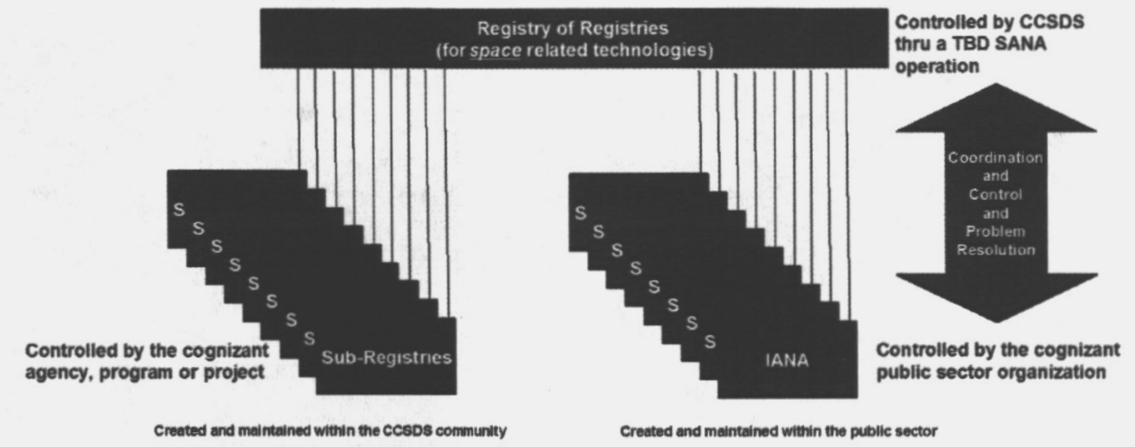

Figure 7, the Public Sector Depiction

\section{What to Register}

The question always arises concerning what to register when we discuss registries. Generally speaking, technologies that have a common and broad application across agencies, systems, applications, technologies and domains may benefit from a registry. This is especially true when they need unique identification. This will include technologies or systems that have multiple agencies and contractors involved in development. There are four categories, which need to be either investigated for registry requirements or assessed for possible adjustment. These categories follow the CCSDS organization process since CCSDS is the only international standards body dealing with space data transport. The four categories are:

Category one (1) is current CCSDS registries, namely SCIDs and SFDU CA.

Category two (2) is the set of protocol identifiers, assigned numbers, port numbers and reserved APIDs that are currently documented within CCSDS approved documents and SCPS protocol numbers including other current deployments like SLE service providers. This would include existing elements e.g. glossary, ground data systems and acronym lists.

Category three (3) is the list of current CCSDS working groups e.g. SM\&C, XML schema and namespaces, and birds of a feather that may require registries and also includes current CCSDS developments.

Category four (4) is the catch all for all other activities which may possess a registries requirement, e.g. information models, reference software, but currently do not fall under CCSDS and/or do not currently operate under a registry.

The technologies in these categories will be looked at a little closer in this section. One item that may not seem to have much significant impact but tends to be a big problem at NASA is the use and reuse of acronyms. A registry of acronyms would help prevent a project from using an acronym that is already in use. This would ensure proper communication between different projects and or centers by ensuring that acronyms are indeed unique. As discussed earlier the SANA registry could take control of a redesigned SCIDs registry to help enforce SCID distributions. Message Bus software would be another technology to consider for a registry. This would handle the types of messages published on the message bus. A developer could then make use of the registry to see which messages that their application could subscribe or publish to the message bus. The IANA could designate a large 
group of IP addresses for the SANA to maintain for spaceflight use or IANA could manage them for SANA. It is easy to see as the deep space network evolves and more elements such as satellites, repeaters, spacecraft elements are added to the network that IP addresses will need to be assigned to this growing number of elements. A registry would be a good way for a user to see what IP address is assigned to a specific element that he/she needs to communicate with. With Ipv6 on the horizon this will become even more important for a registry to control these new IP addresses for space elements. XML schema, which addresses certain commands or telemetry, could be kept in a registry. Users wanting to develop tools to make use of these types of commands and telemetry would be able to access the registry and see what format of XML schema to use. This would make interoperability much easier since all users would be required to use the same schema for certain commands and telemetry. Without this type of registry there would be no easy way to prevent multiple users from developing their own unique XML schemas for the same telemetry and commands making interoperability between tools much less certain.

\section{Registry Information Access}

There must be easy access by operations and development personnel to registry information. Obviously current technology is adequate to facilitate broad access. Depending on the technology and the responsible registry authority, it is anticipated that access will be via a single authoritative access point which either possess the information or points to it. It should be pointed out that the registries described in this paper will not contain user data e.g. act as a repository or archive. There can be many implementations for the access but one that holds promise is to create a portal for entry into a registry of registries. From this portal all registered information with regards to space operations would be available. Across the top tabs of the portal would be the disciplines for instance ground control systems - commanding management, telemetry management. If a user chooses command management then all associated registry information would be presented. Along the side would be lists of services available to the specific choice made in this case Command Management. These services could range from those available to anyone like voice and video conferencing to very discipline specific authoritative information like navigation registries. One of the main benefits is that everything presented in the portal is authoritative. The ideal setup is to configure a registry of registries that will allow one to be able to locate, get details about, and make use of any resource located in our registry through structured metadata. In this registry configuration, there will be four important components: a full searchable registry, local searchable registry, local publishing registry, and a registry of registries. Of course, standards and protocols must be defined so that different registry services will be able to interoperate. Therefore, the architecture and organization of the registry must allow all others to have access. 'Two important standards that will help define the registry are interface specification and XML schemas. The interface specification is the agreement amongst different parties for defining the search interface. XML schemas formally define the various resource types and allow incoming and outgoing messages to be automatically verified for correctness of content and structure. XML and its related technologies will be a great choice for protocol. Not only is XML a standard way of formatting information, but it also provides XML parsers, namespaces and schemas. Registry commands will provide an interface to manage the registry files. Our registry setup does not have to be a Service Oriented Architecture (SOA)-based service registry. But a SOA can provide some advantages. SOAs are frameworks for reusability.

\section{Management and Control}

Overall management and control of the registry process should not be resident in any single national entity but should be controlled with broad international involvement. Currently there is only one organization that traverses and involves most civilian national space agencies. This is the CCSDS. This organization has been in existence for well over 25 years. It has a management structure that enables involvement on the international level. Figure 1 above shows the organizational structure of CCSDS and a list of member and observer agencies are in Appendix A.

The CCSDS uses working groups staffed by personnel from the various member agencies. Birds of a Feather (BoFs) are precursors to working groups. $\mathrm{A} \mathrm{BoF}$ defines the charter, estimated resources and schedule for a working group. After the CESG and CMC review, and if approved, then a working group is formed. The subject, as the list indicates, varies widely. Appendix B lists the current CCSDS working groups and birds of a feather.

\section{Conclusion}

The SANA will provide a registry service that will greatly enhance current and future space operations. The amount of services that will be needed to get back to the Moon and eventually Mars will be very extensive. The SANA will provide a means to register these services and control the use of these services. 


\section{References}

[1 "Consultative Committee for Space Data Systems (CCSDS)," [www.ccsds.org. Accessed 5/18/06.]

[2]"Dictionary.com,"'[www.dictonary.reference.com. accessed 5/18/06.]

[2]"CCSDS Global Spacecraft Identification Field Code Assignment Control Procedures. Blue Book. Issue 3, CCSDS 320.0-B-

3" April 2003. [http://public.ccsds.org/publications/archive/320x0b3.pdf. Accessed 5-16/2006.]

[3] "Space Link Identifiers Blue Book. Issue 2, CCSDS 135.0-B-2. November 2005.

[http://public.ccsds.org/publications/archive/135x0b2.pdf. Accessed 5/20/2006.]

] "NISN Services Document," Marshall Space Flight Center, Alabama, 2005. [http://nisn.msfc.nasa.gov. Accessed 5/16/2006.] 


\section{Appendix A, List of CCSDS Member and Observer Organizations ${ }^{6}$}

Member Agencies

- Agenzia Spaziale Italiana (ASI)/Italy.

- British National Space Centre (BNSC)/United Kingdom.

- Canadian Space Agency (CSA)/Canada.

- Centre National d'Etudes Spatiales (CNES)/France.

- Deutsches Zentrum für Luft- und Raumfahrt e.V. (DLR)/Germany.

- European Space Agency (ESA)/Europe.

- Russian Federal Space Agency (FSA)/Russian Federation.

- Instituto Nacional de Pesquisas Espaciais (INPE)/Brazil.

- Japan Aerospace Exploration Agency (JAXA)/Japan

- National Aeronautics and Space Administration (NASA)/USA.

Observer Agencies

- Austrian Space Agency (ASA)/Austria.

- Central Research Institute of Machine Building (TsNIIMash)/Russian Federation.

- Centro Tecnico Aeroespacial (CTA)/Brazil.

- Chinese Academy of Space Technology (CAST)/China.

- Commonwealth Scientific and Industrial Research Organization (CSIRO)/Australia.

- Communications Research Laboratory (CRL)/Japan.

- Danish Space Research Institute (DSRI)/Denmark.

- European Organization for the Exploitation of Meteorological Satellites

(EUMETSAT)/Europe.

- European Telecommunications Satellite Organization (EUTELSAT)/Europe.

- Federal Service of Scientific, Technical \& Cultural Affairs (FSST\&CA)/Belgium.

- Hellenic National Space Committee (HNSC)/Greece.

- Indian Space Research Organization (ISRO)/India.

- Institute of Space and Astronautical Science (ISAS)/Japan.

- Institute of Space Research (IKI)/Russian Federation.

- KFKI Research Institute for Particle \& Nuclear Physics (KFKI)/Hungary.

- MIKOMTEK: CSIR (CSIR)/Republic of South Africa.

- Korea Aerospace Research Institute (KARI)/Korea.

- Ministry of Communications (MOC)/Israel.

- National Oceanic \& Atmospheric Administration (NOAA)/USA.

- National Space Program Office (NSPO)/Taipei.

- Space and Upper Atmosphere Research Commission (SUPARCO)/Pakistan.

- Swedish Space Corporation (SSC)/Sweden.

- United States Geological Survey (USGS)/USA 


\section{Appendix B, List of CCSDS Working Groups (WG) and Birds of a Feather (BoFs) ${ }^{7}$}

System Architecture WG

Information Architecture WG

Security WG

Space Assigned Numbers Authority WG

Space/Ground Interoperability Architecture BoF

XML Standards \& Guidelines SIG

Delta-DOR SIG

Data Archive Ingestion WG

Navigation WG

Information packaging \& Registries WG

Spacecraft Monitoring and Control WG

Reference Model \& Concept WG

Service Management WG

Cross Support Transfer Service WG

Onboard Bus LAN WG

Time Critical Onboard Network Services WG

Time Critical Onboard Application Services WG

Onboard Plug and Play BoF

Onboard Transducer System BoF

Wireless BoF

RF Modulation WG

Space Link Coding and Synchronization WG

Data Compression WG

Space Link Protocols WG

Telecommand Channel Coding WG

Ranging WG

Proximity-1, Build-2 WG

High Rate Uplink WG

Long Erasure Codes BoF

CFDP Interoperability Testing WG

Packet Protocol WG

Cislunar WG

Asynchronous Message Service WG

IP over CCSDS Space Links WG

Mars Communication Profile $\mathrm{BoF}$ 

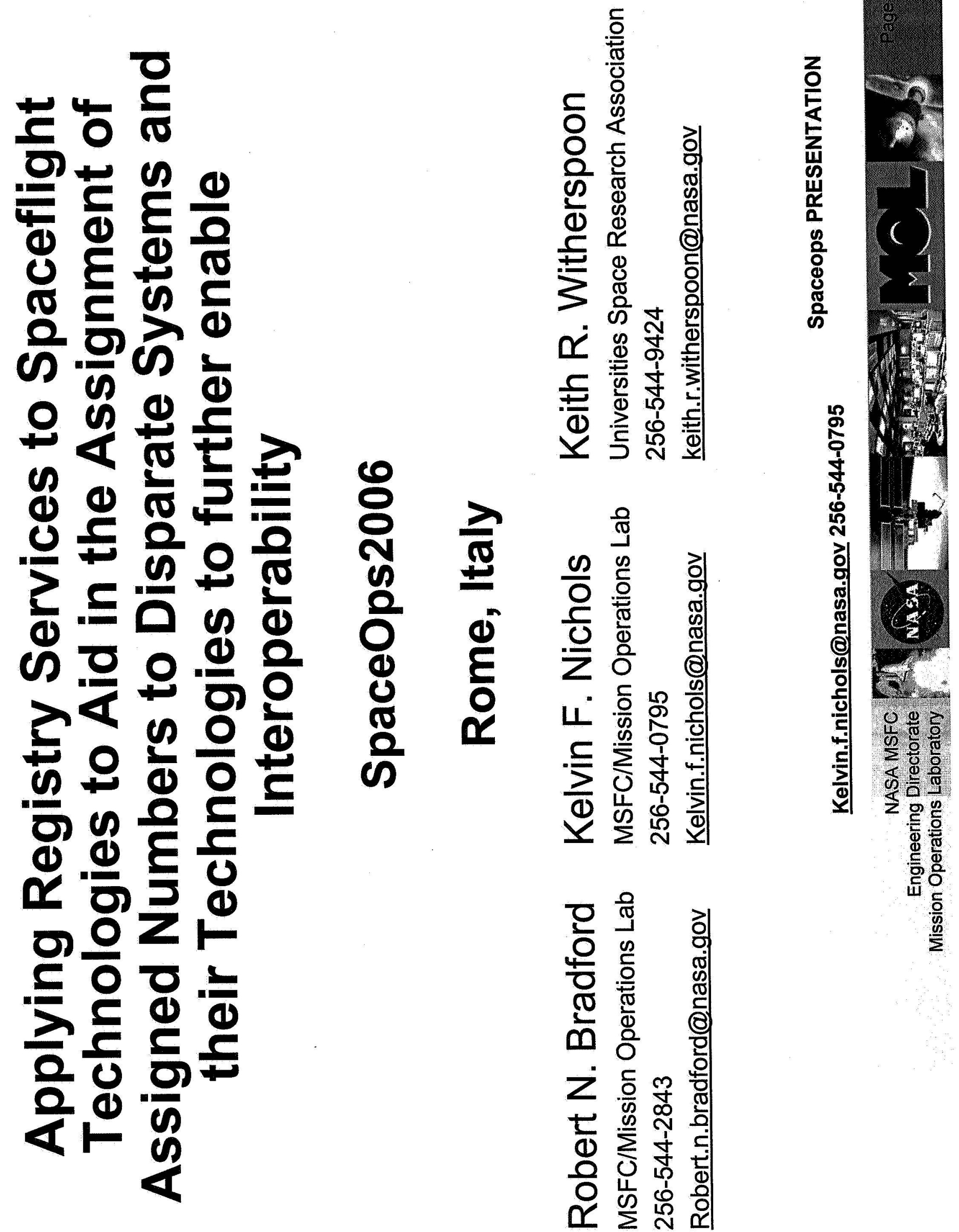

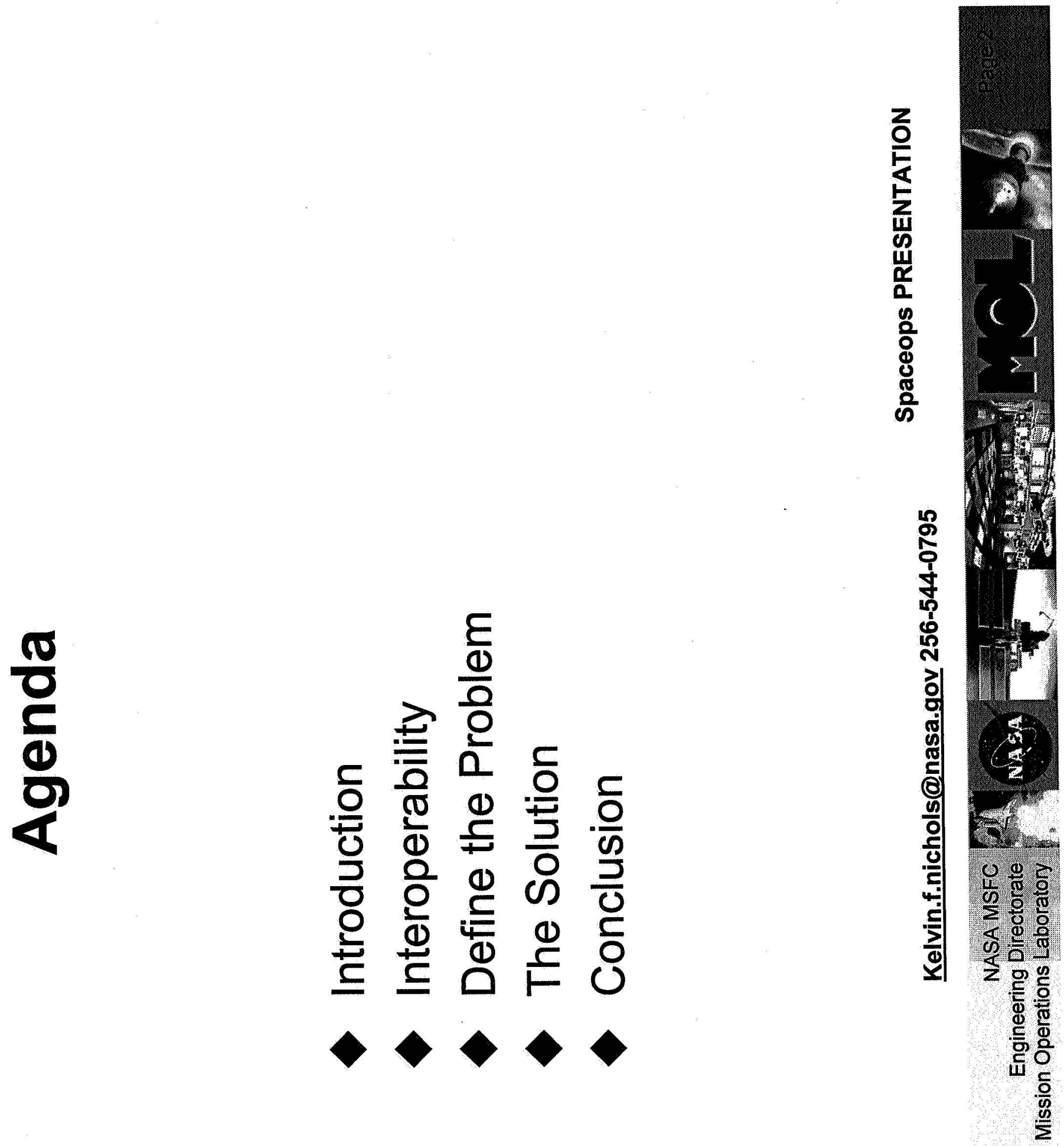


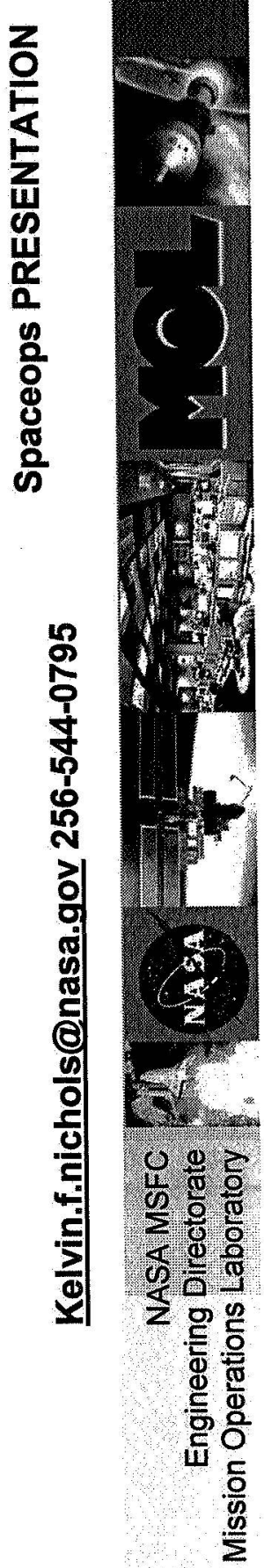




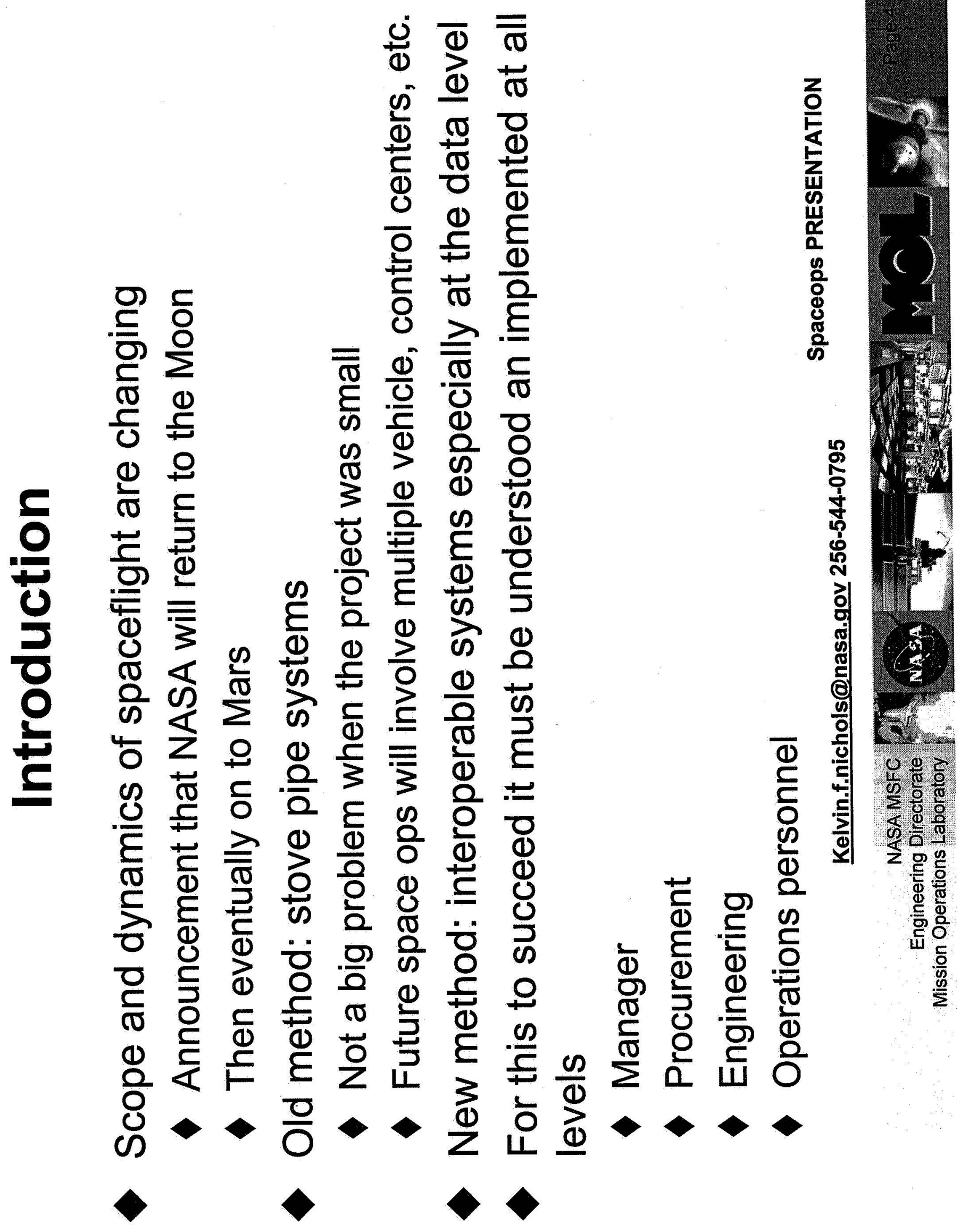




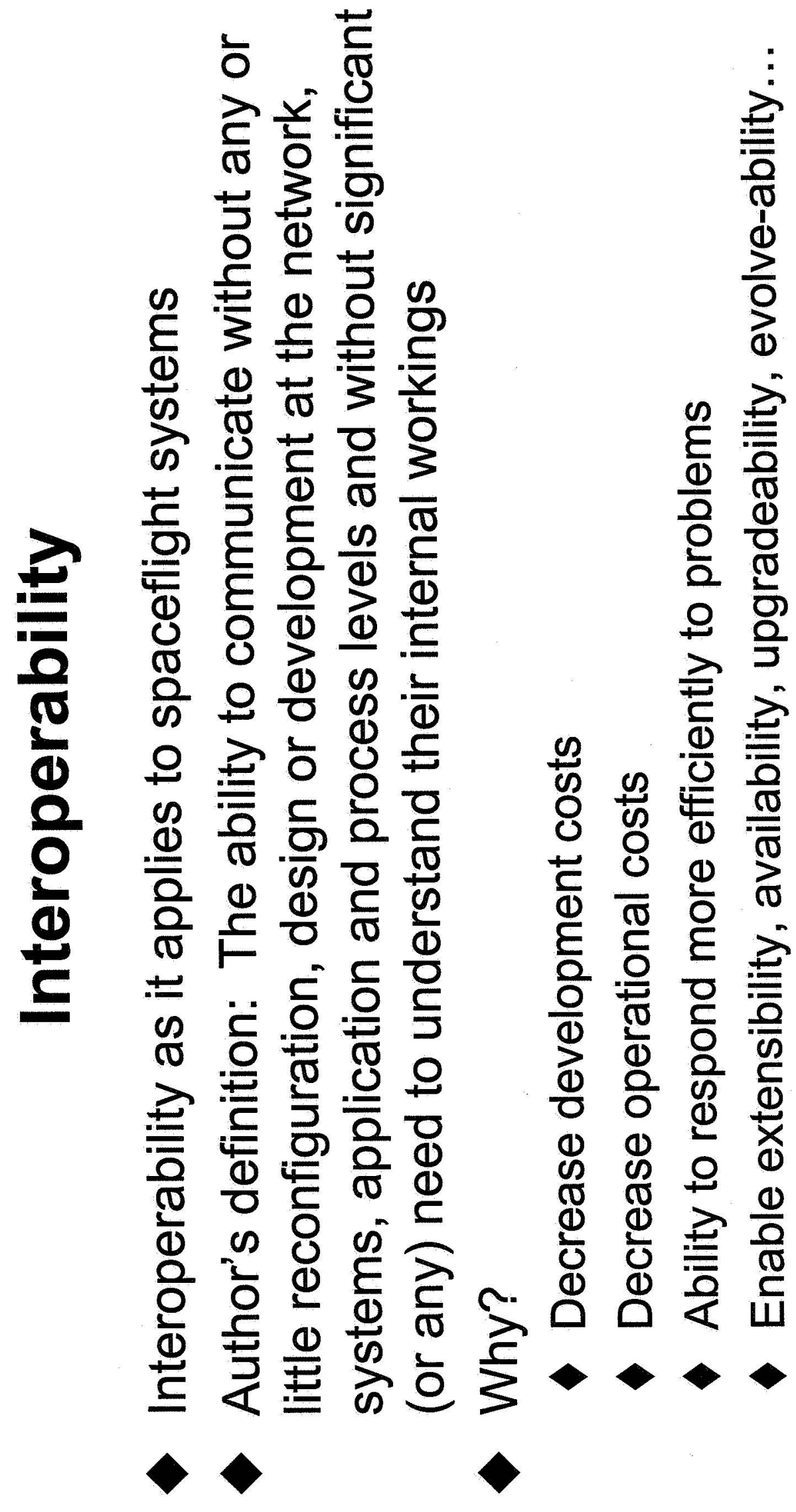




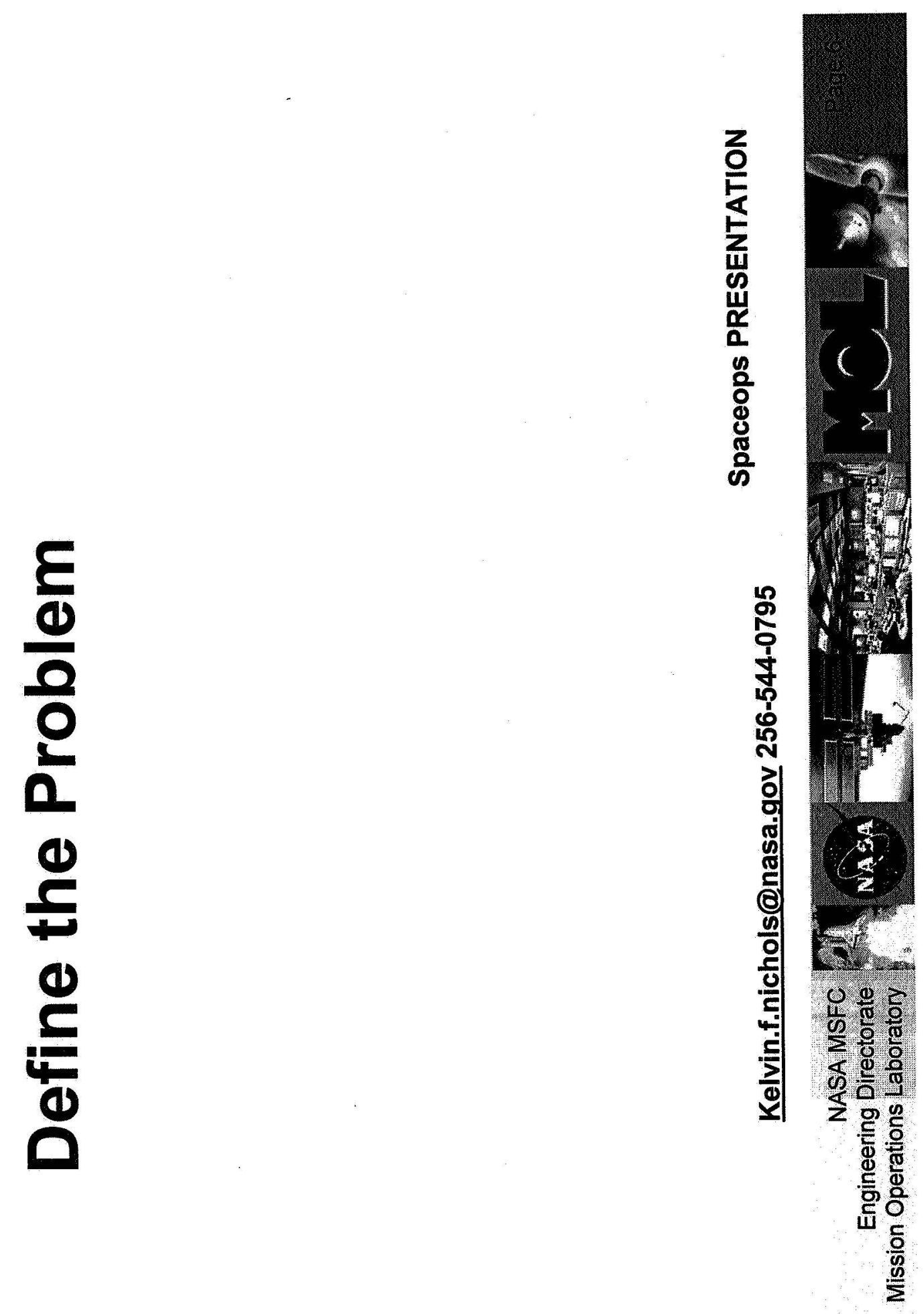




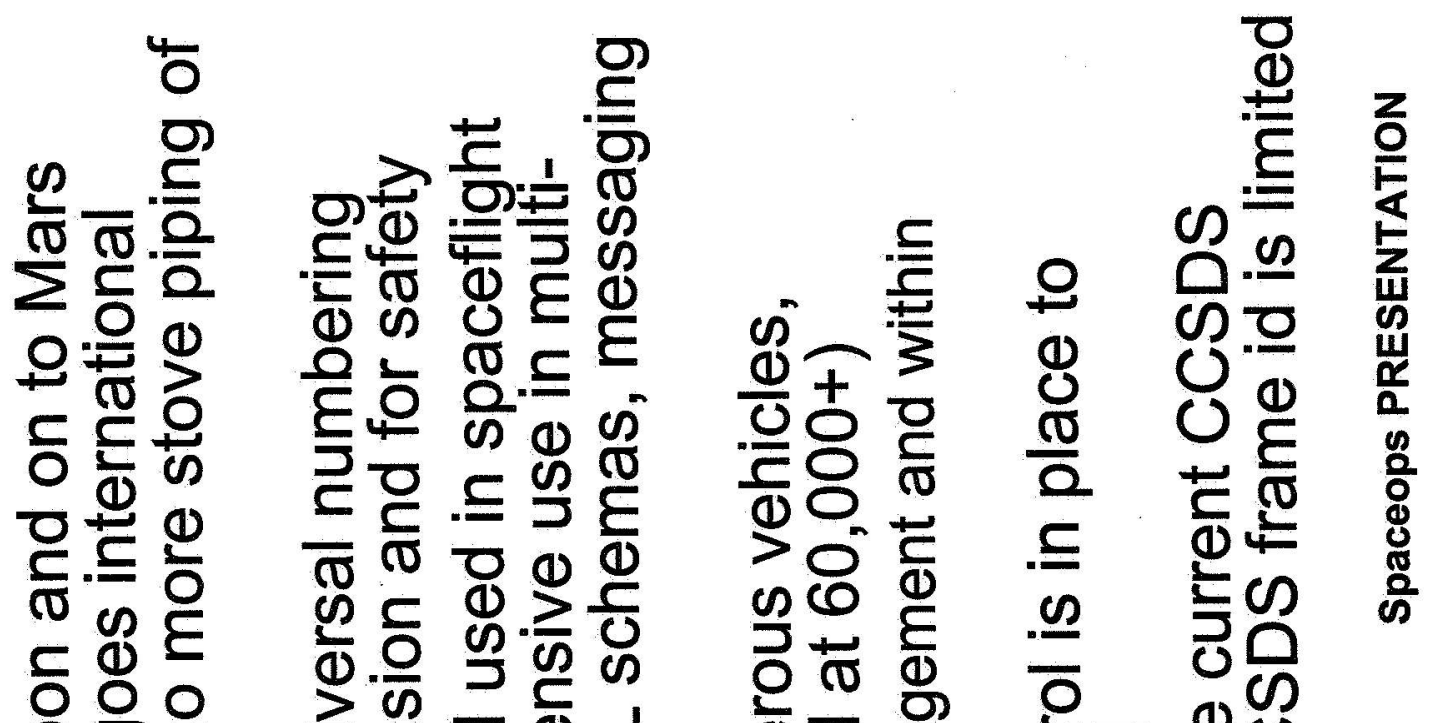

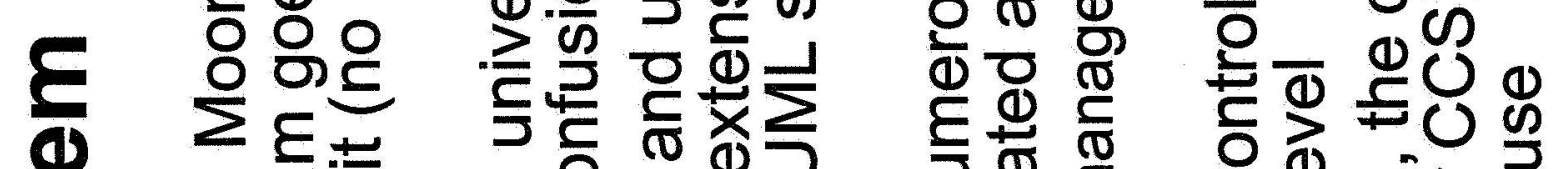

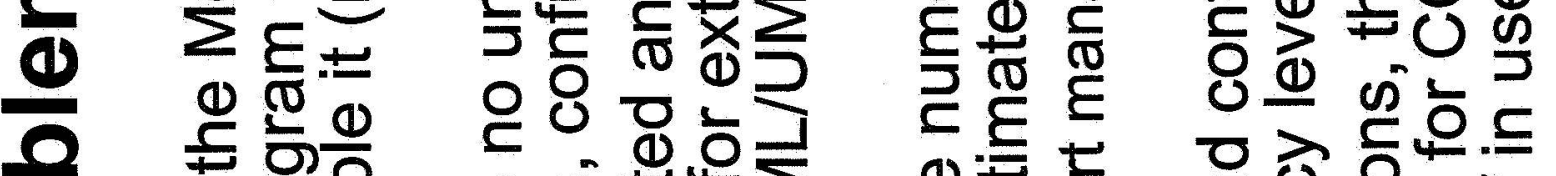

凹

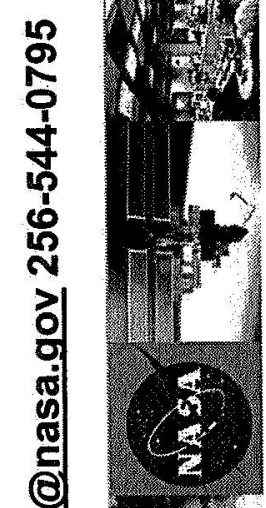

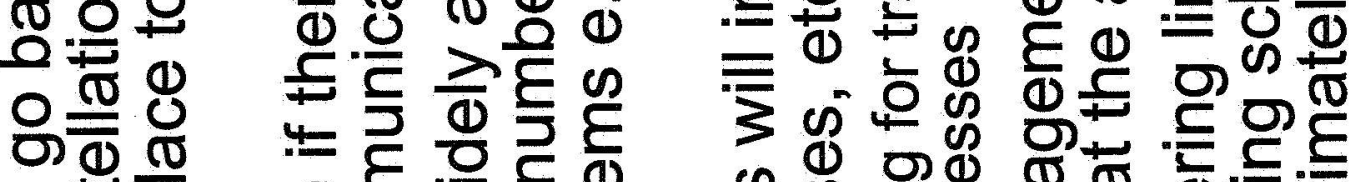

유을

()

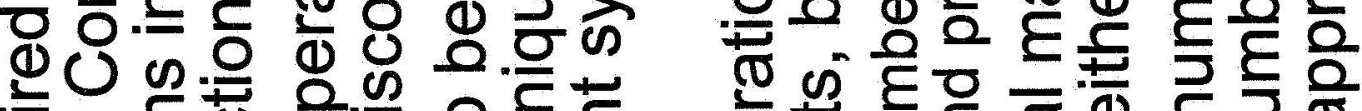

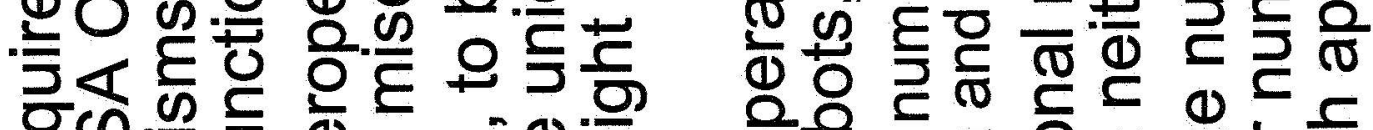

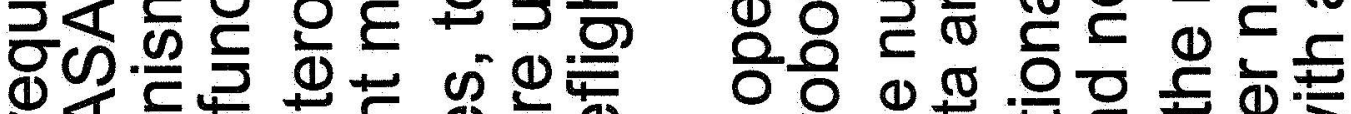

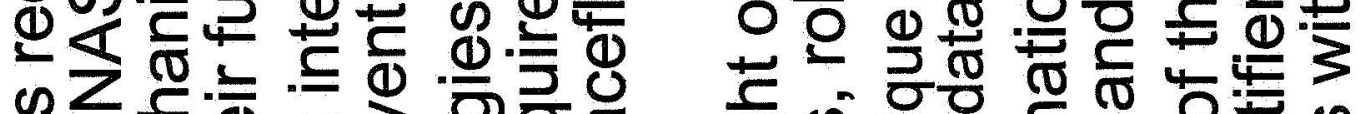

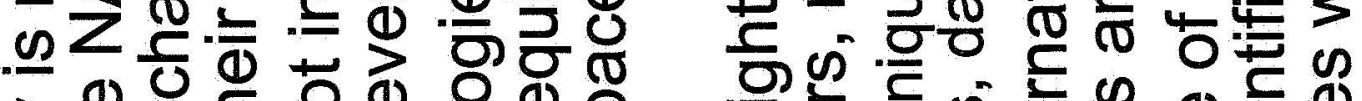

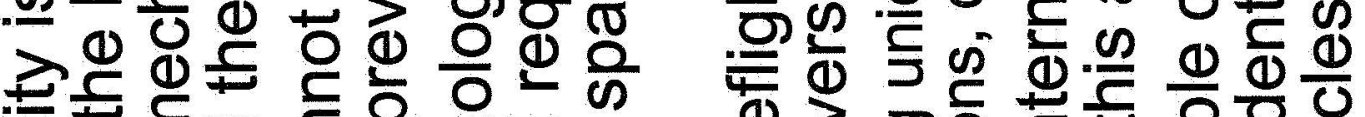

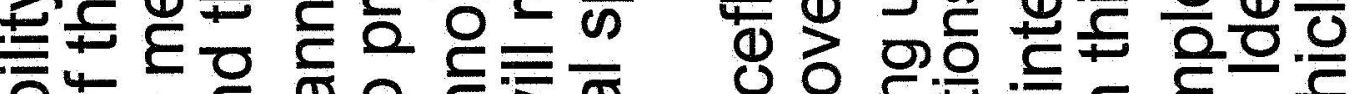

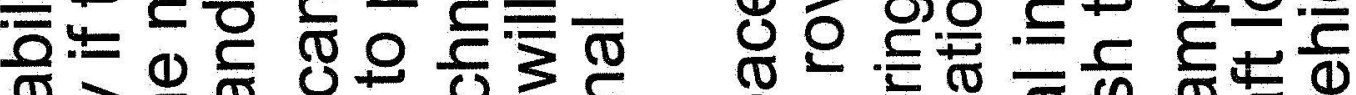

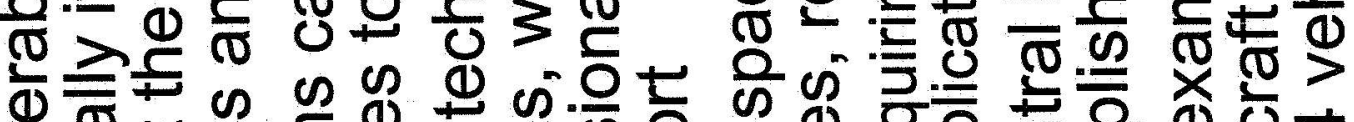

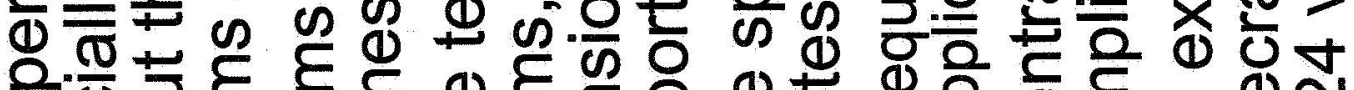
응

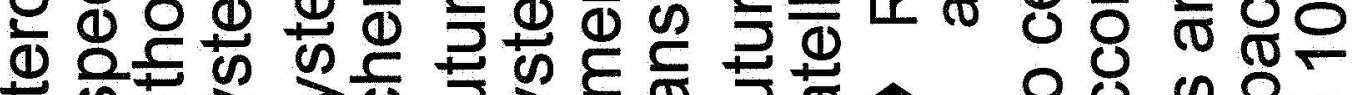

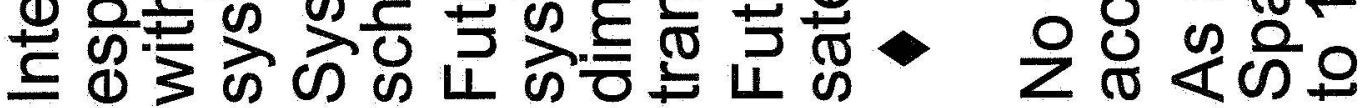




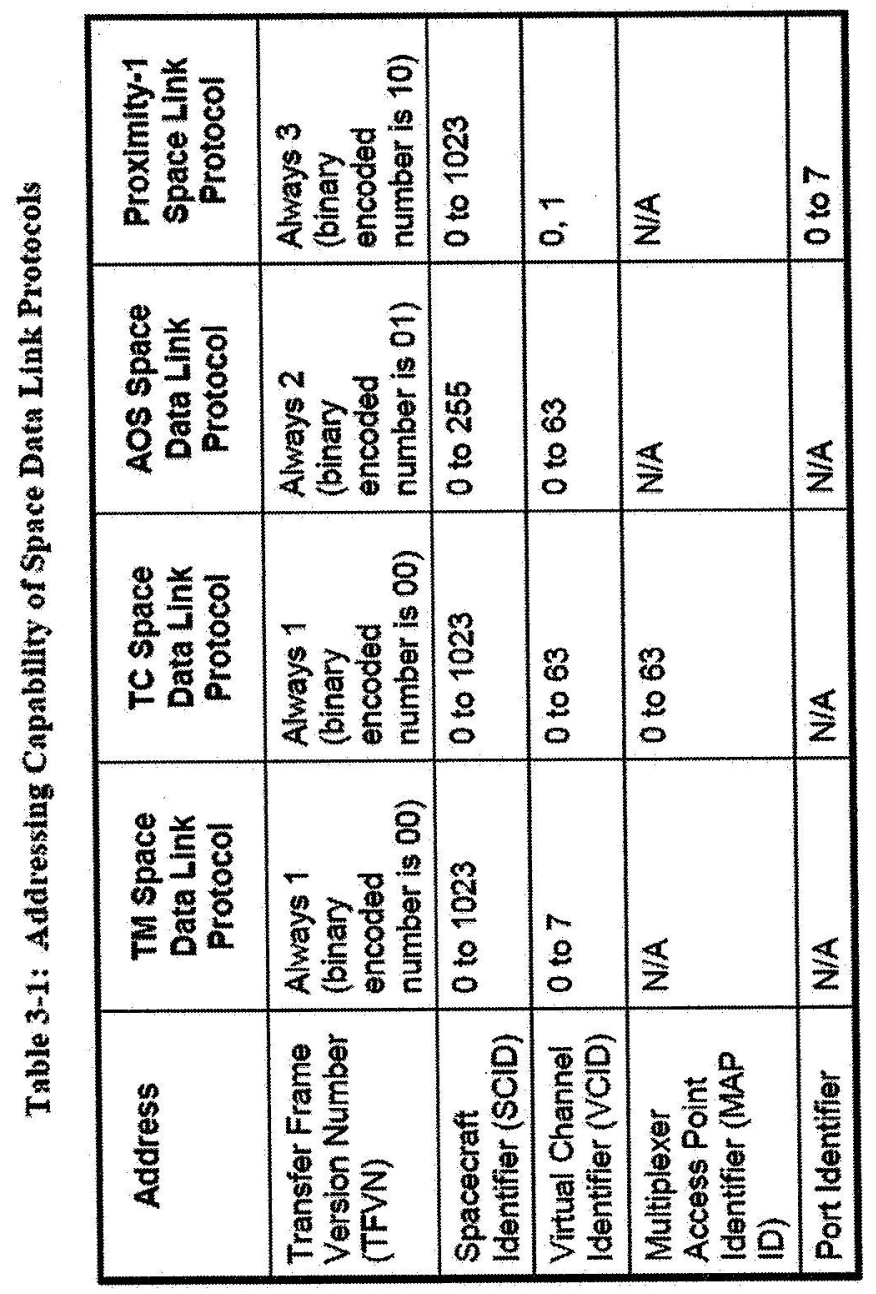

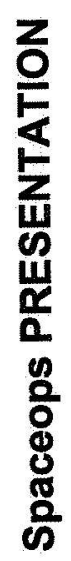

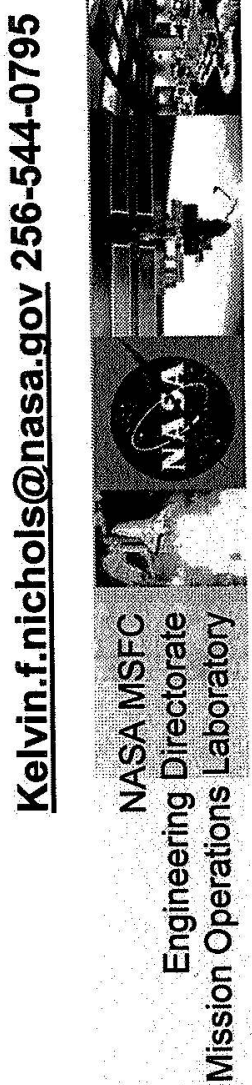




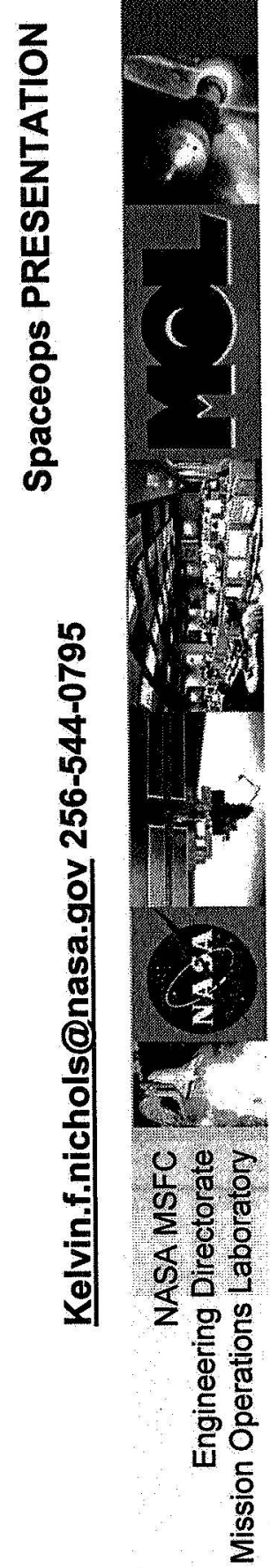


ఫั

の

$\underline{\mathscr{E}}$

$\frac{1}{c}$

응 는 등 엉

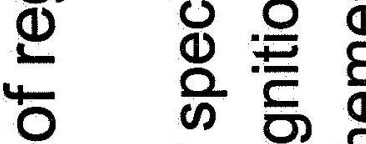

ஸ $\bar{\delta}$

ठ্ঠ

兵 ठ্ঠ

0 동 응

( )

()

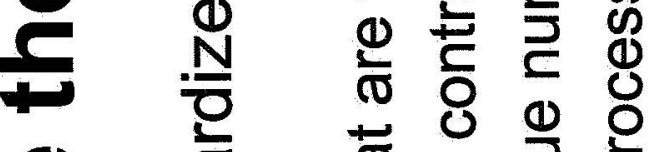

해

을 을.을 을을

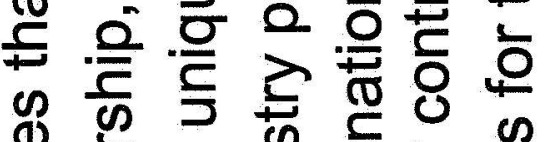

运

क

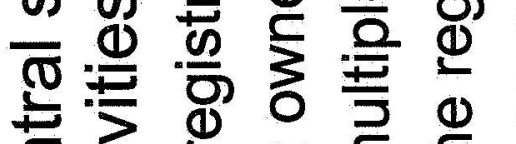

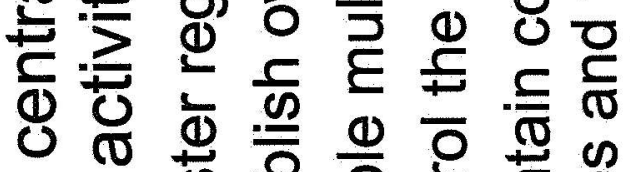

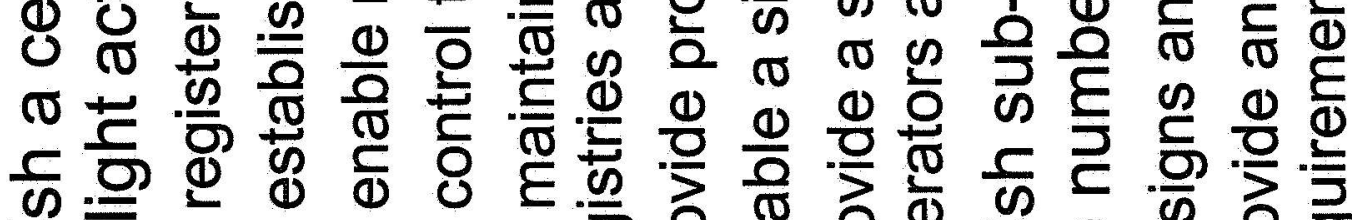

क⿻

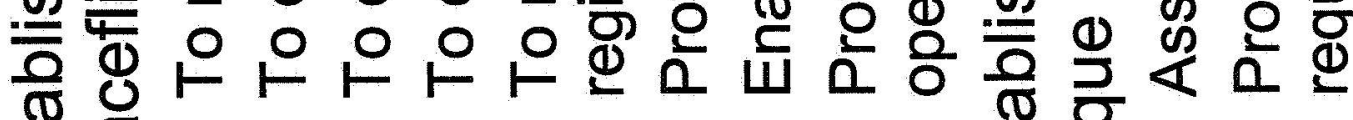

त्త

ய क<smiles>[C-]#CC#C[Se]</smiles>

त 0

$\subsetneq$

ल ब

응 을 층

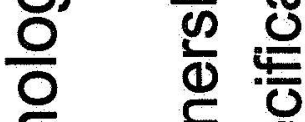

ᄃ

य) $2 \geq$

क :

은 인

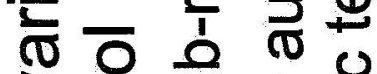
는

这 क क 0 잉 के

은

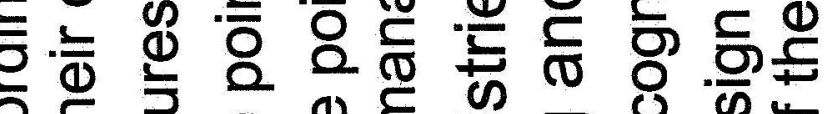

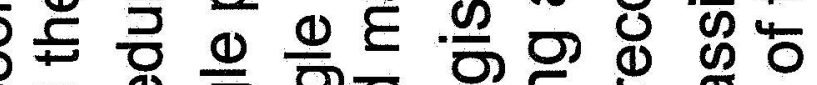
히응

$\frac{\infty}{9}$ 


\section{}

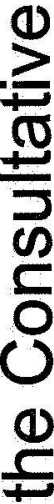

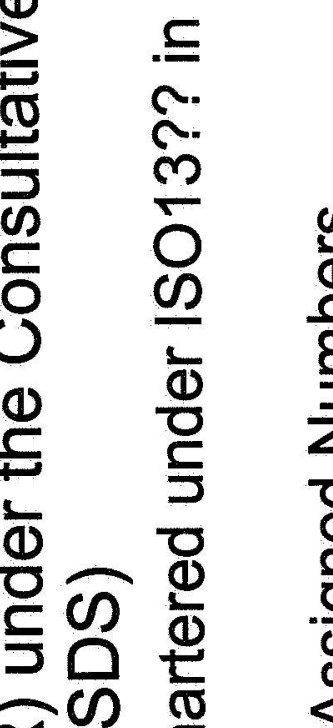

.

.

.

$\omega$

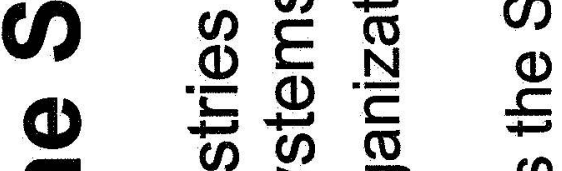

둥ㅁㅇ

(1)

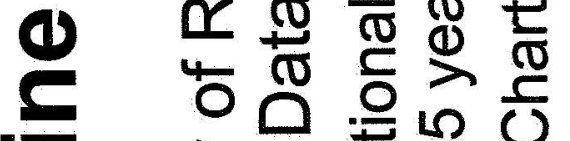

420 市

(1)

ด

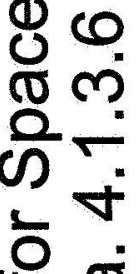

4

$\Phi$ ธั

Q

$\equiv=\frac{1}{\pi}$

ह ํㅇㅇ

है

ง

(1) 은

$\geqq \frac{\bar{\sigma}}{(1)}$

$\pi>$

$\pm 0$

कㄴำ

ㅇํㅇㄹㅛ

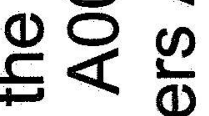

ㅇํㅇ

๘

马UZ

정

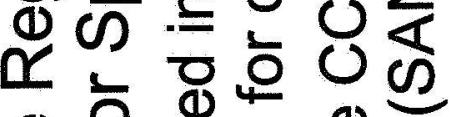

0 은

토 을 을 을

凹

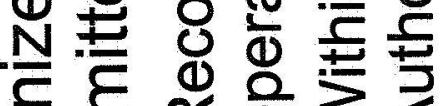

들 잉 응

ช $\frac{1}{0}$

○ी
क $\frac{\bar{d}}{\mathrm{~s}} \cdot \frac{\bar{\sigma}}{\mathrm{s}}$

(1)

䨌文

ठิ

Uु

ํํำ 옹 $\frac{\omega}{9}$

8

웡몬

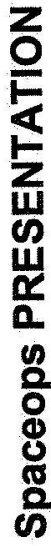

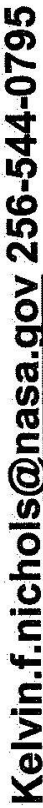

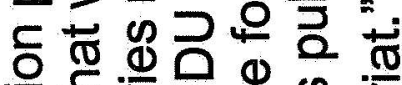

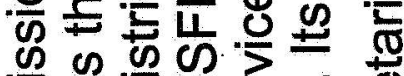

.

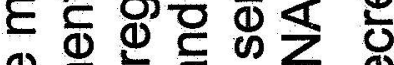

ه

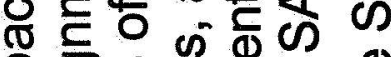

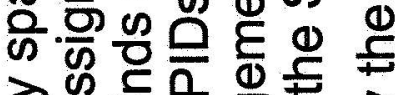

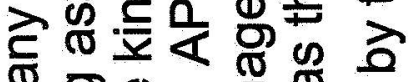

స్

$\Sigma . \sqsubseteq \Phi$ 可

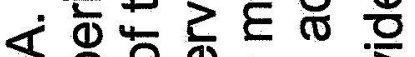

之워원을 은

⿰ 亏은

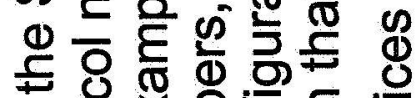

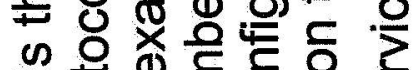

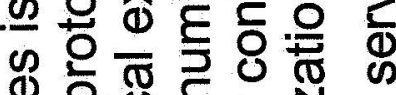

¿

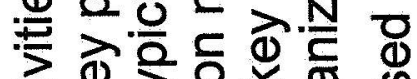

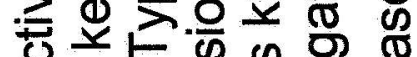

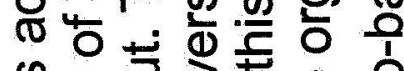

n 드워

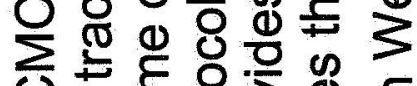

응유 동

Ф 엉응응윽

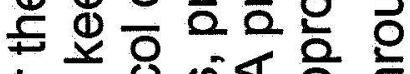

는

등흐웡

চ

屯

面品 \&

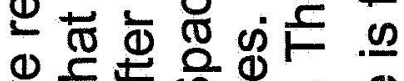

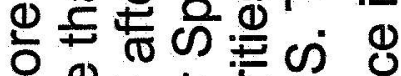
ठ워유

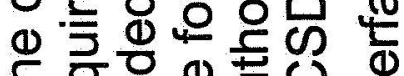

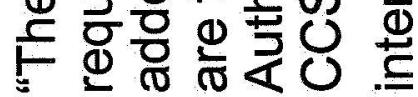

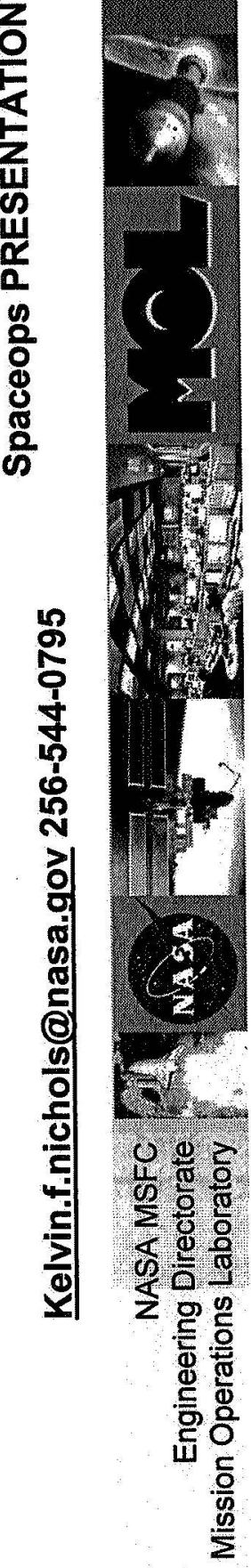




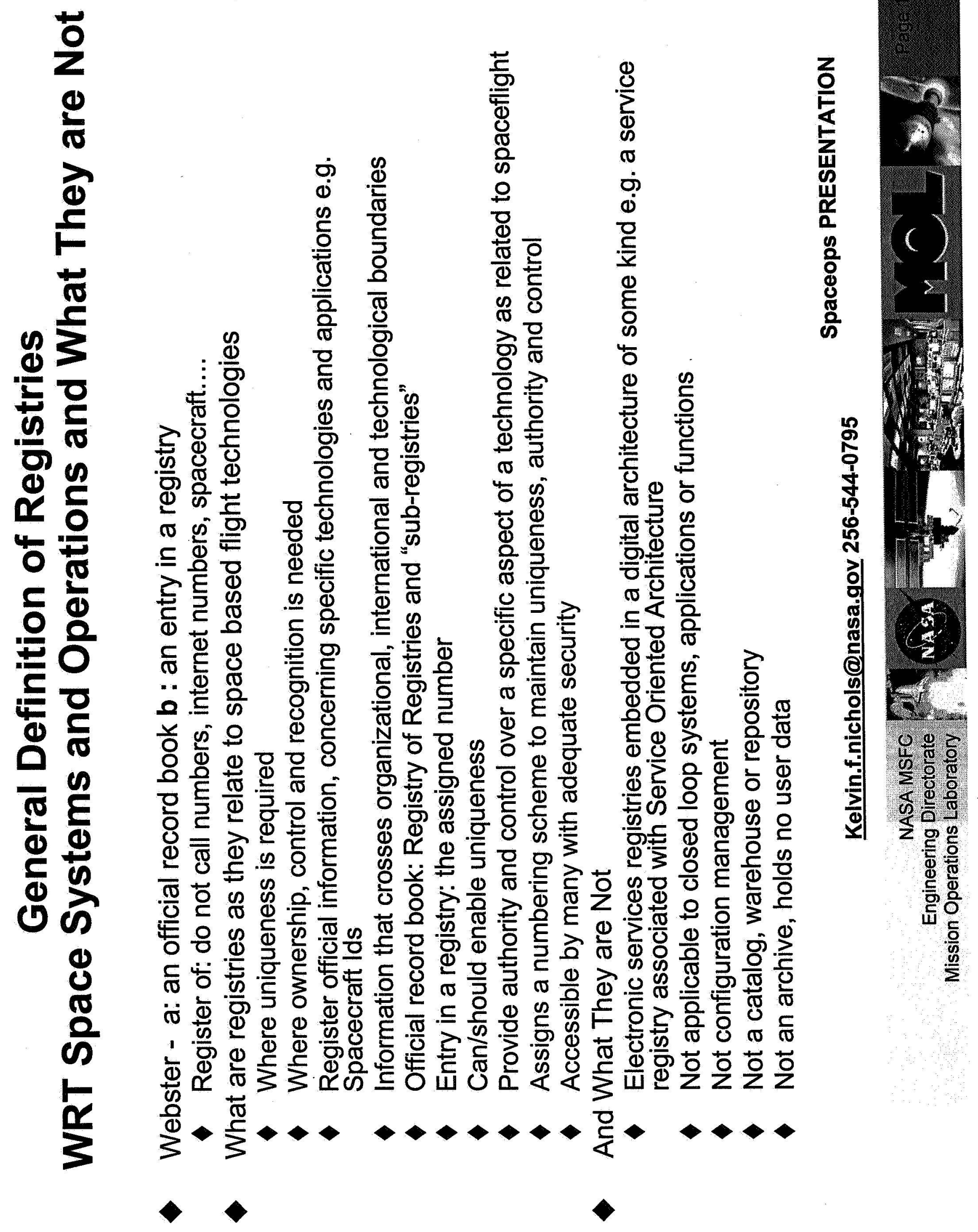




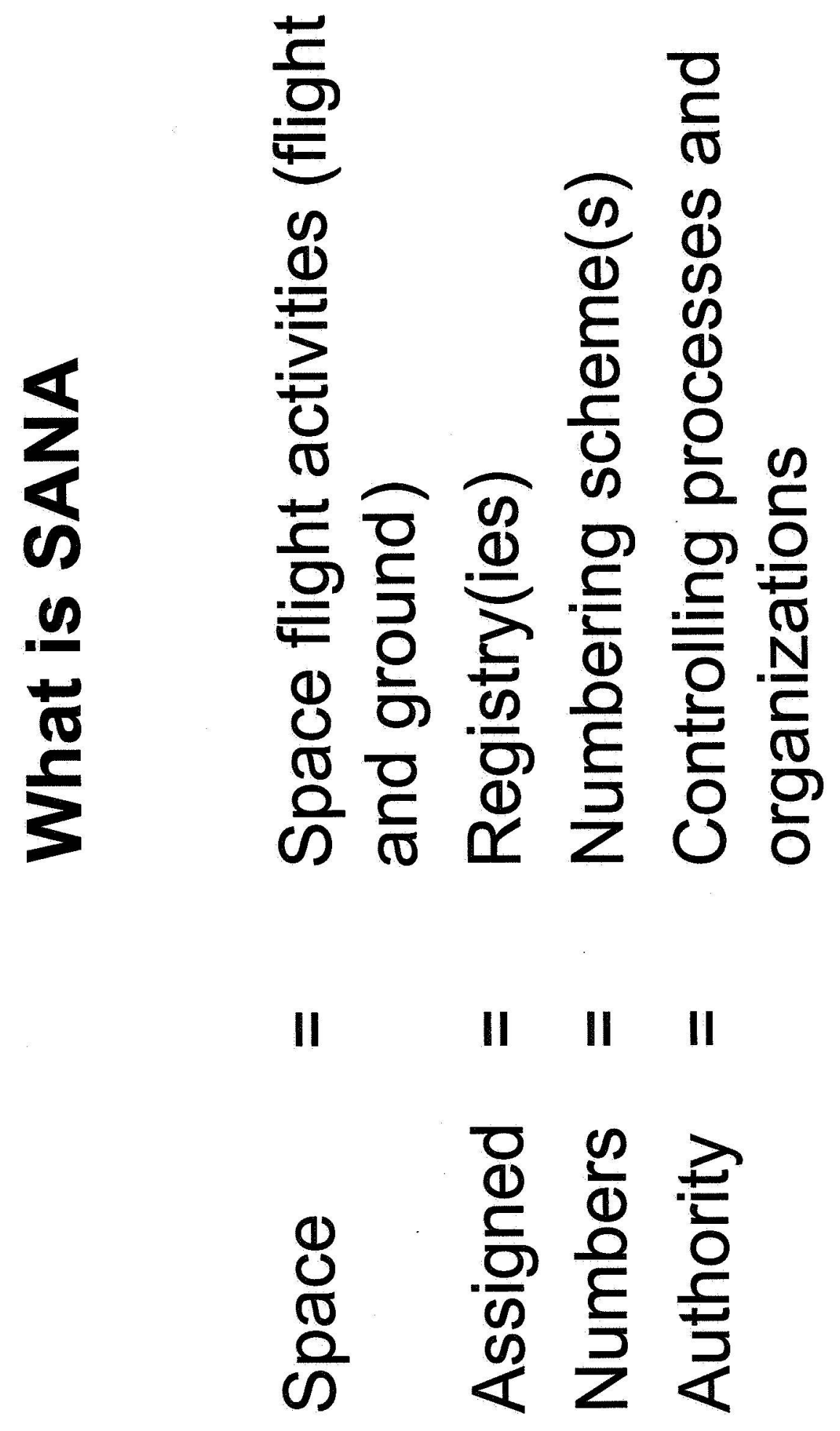




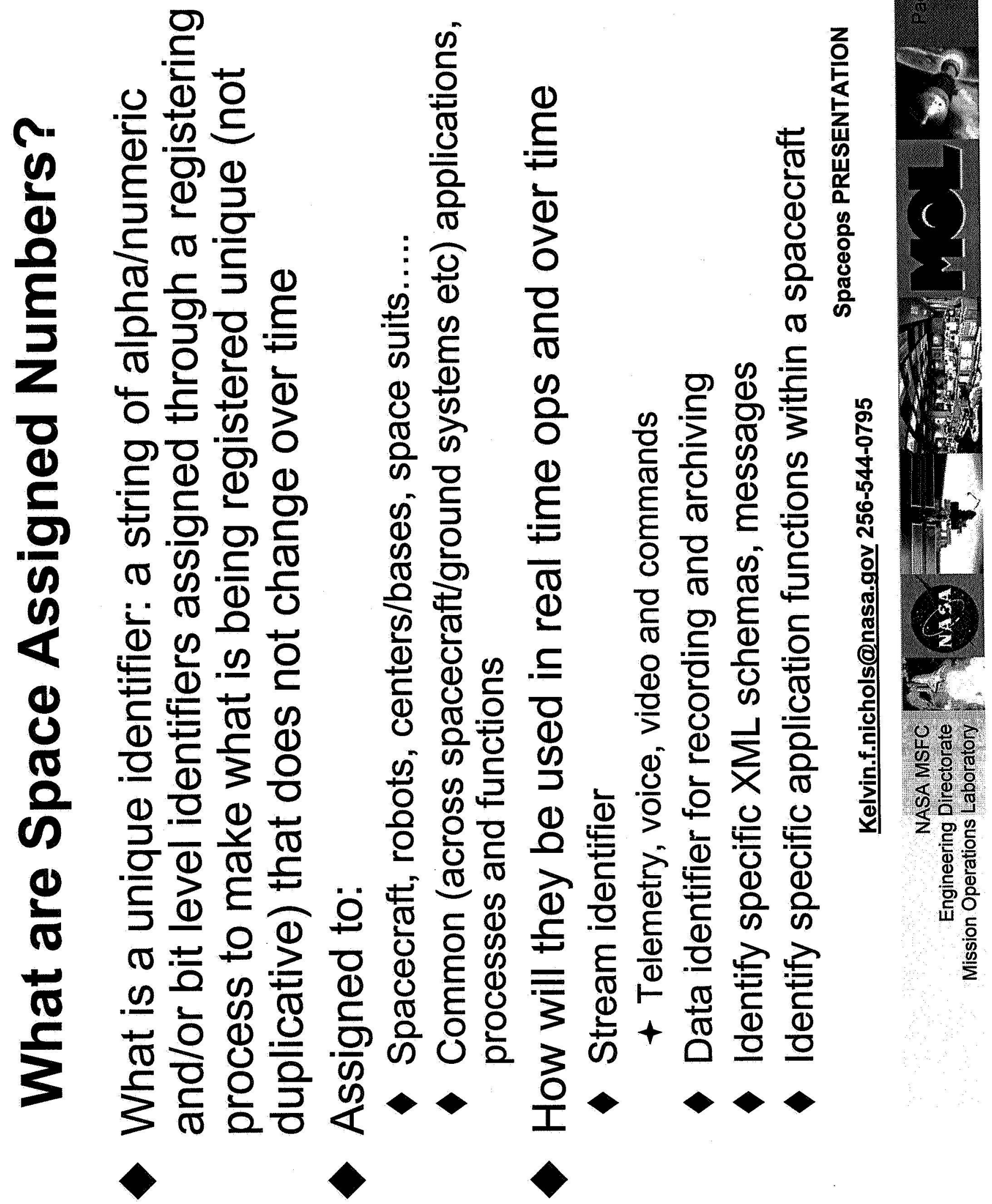




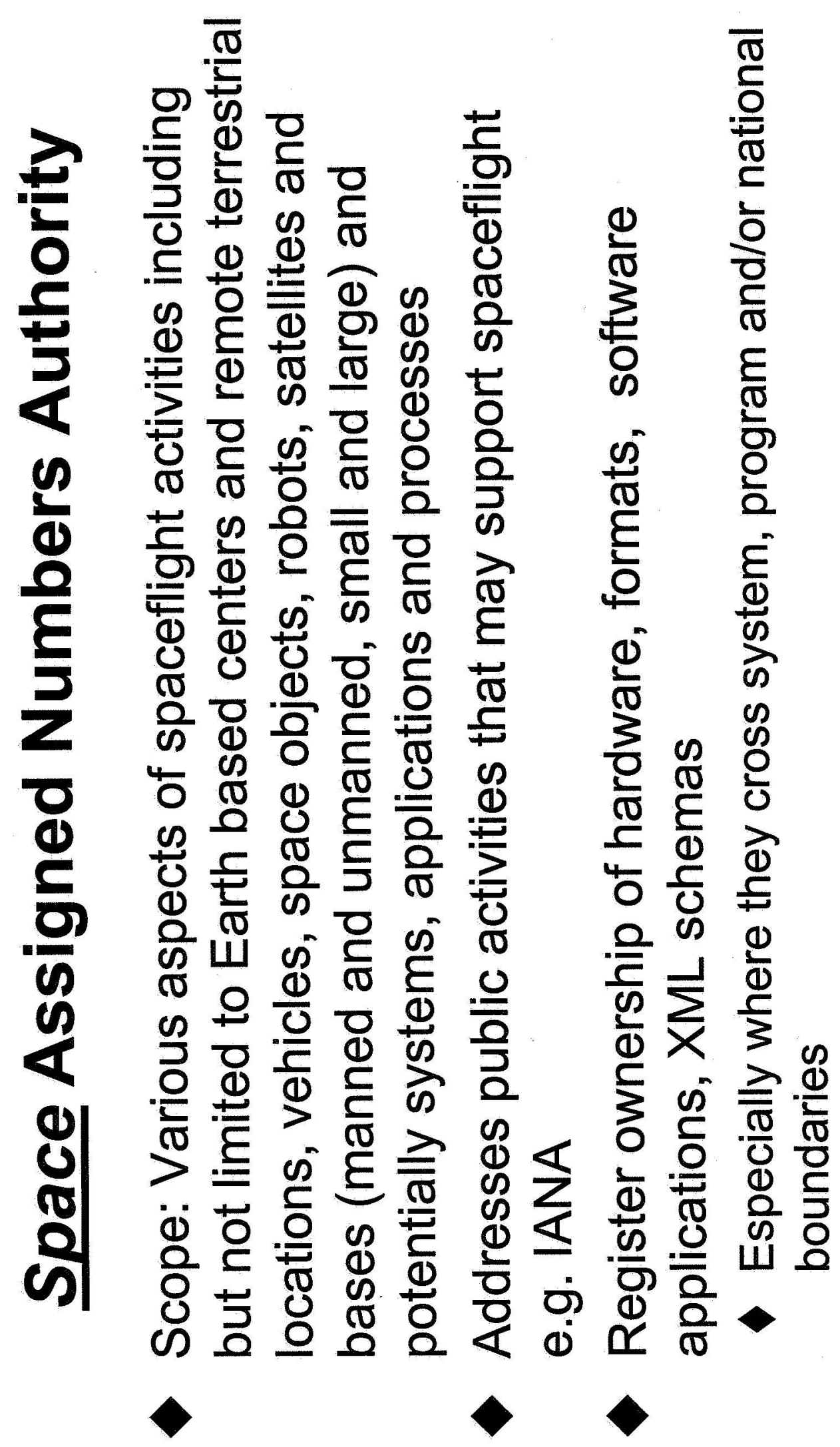

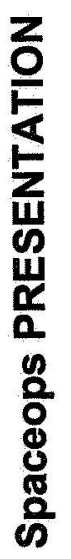

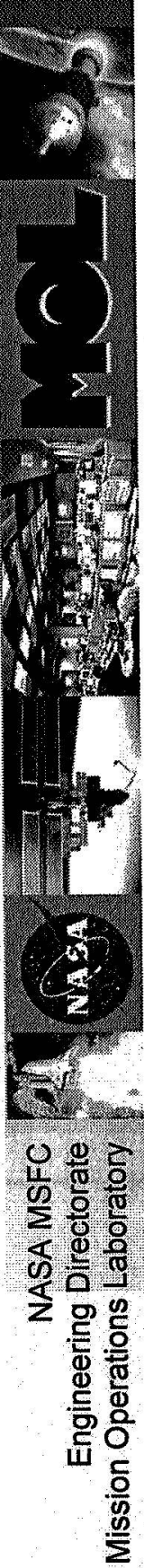




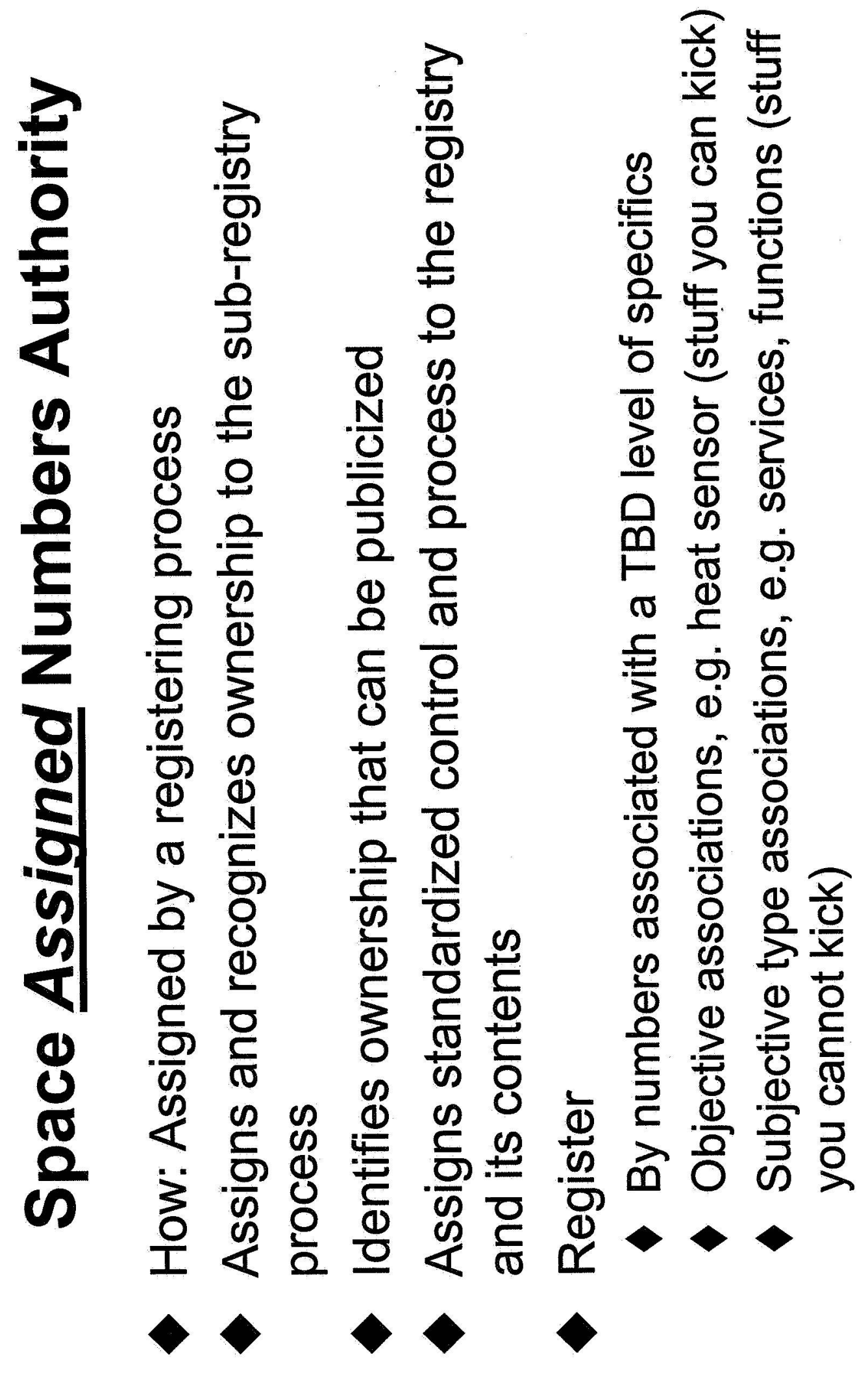

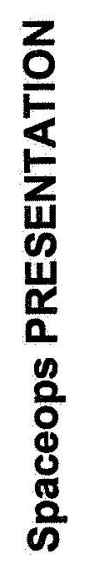

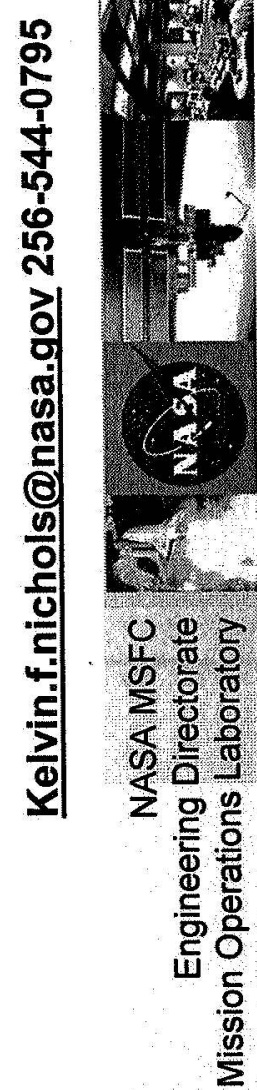




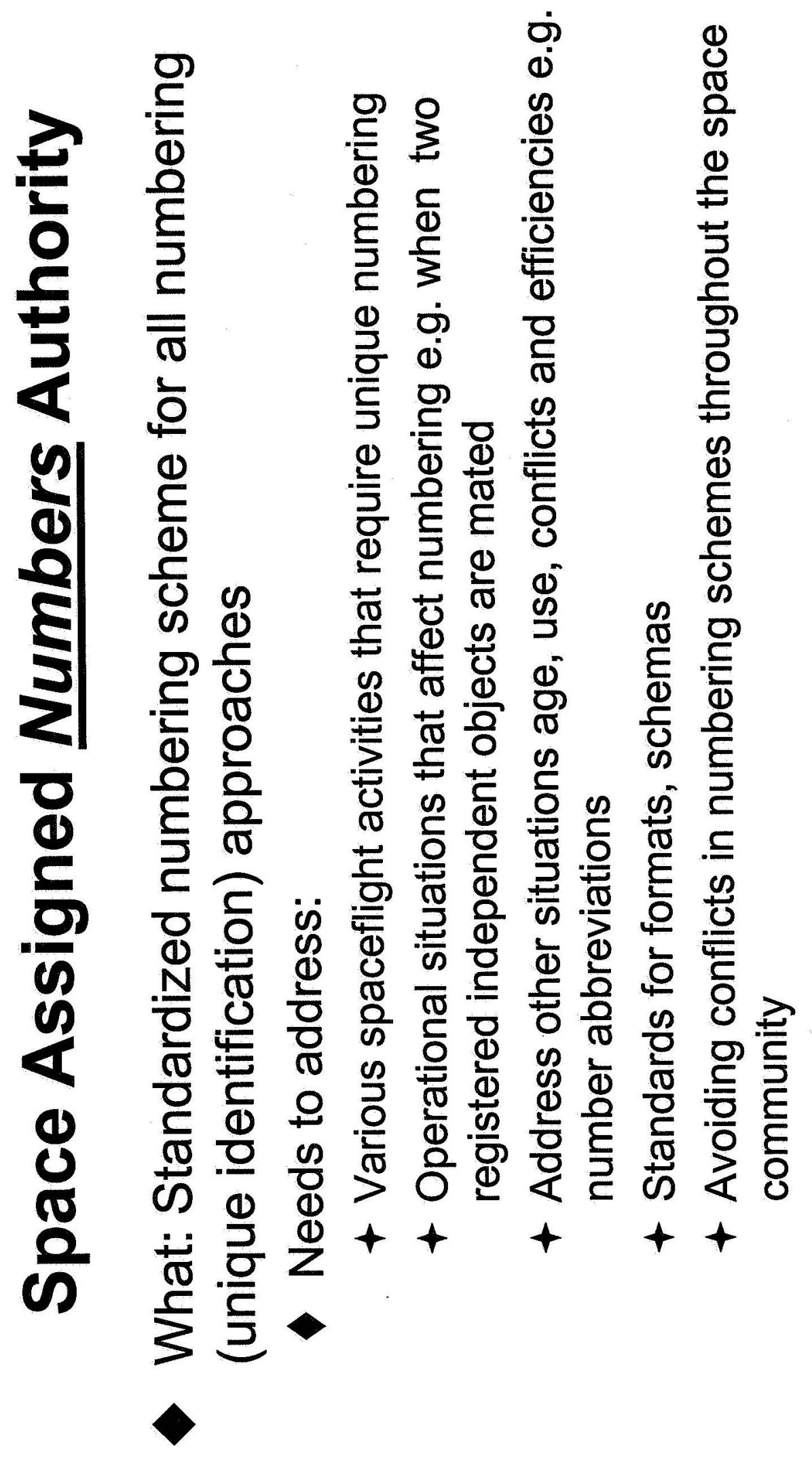

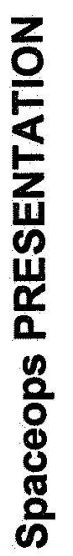

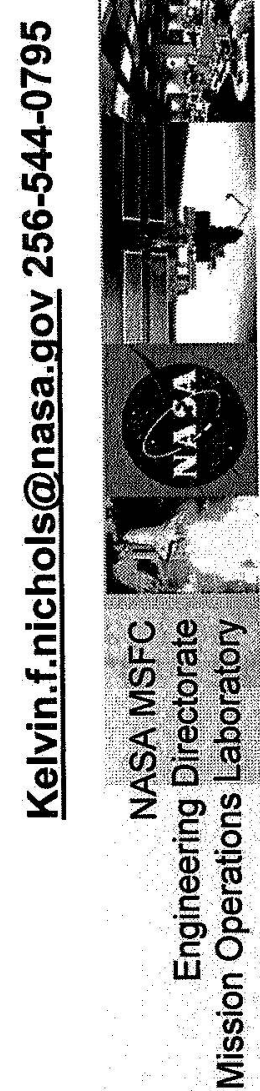




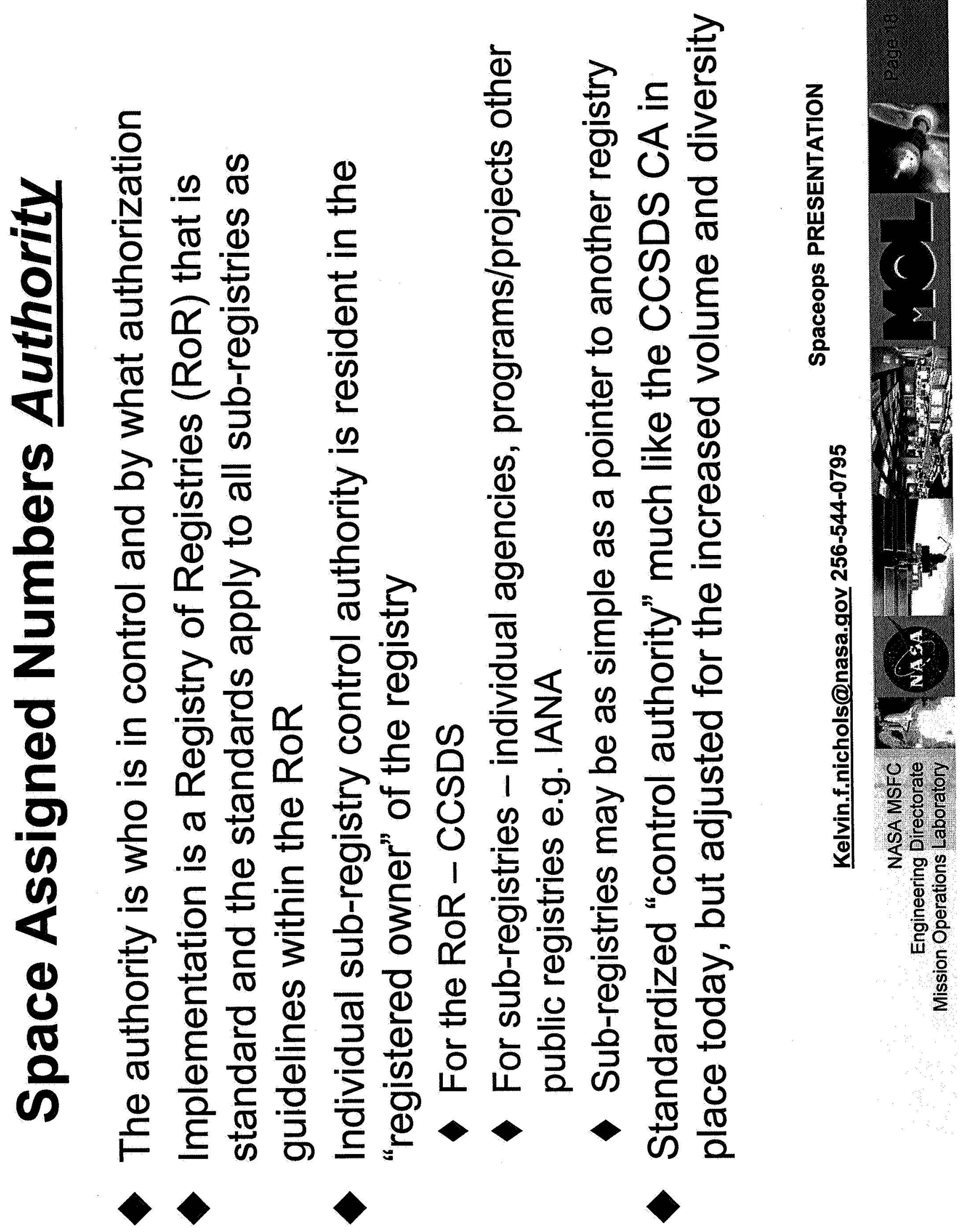




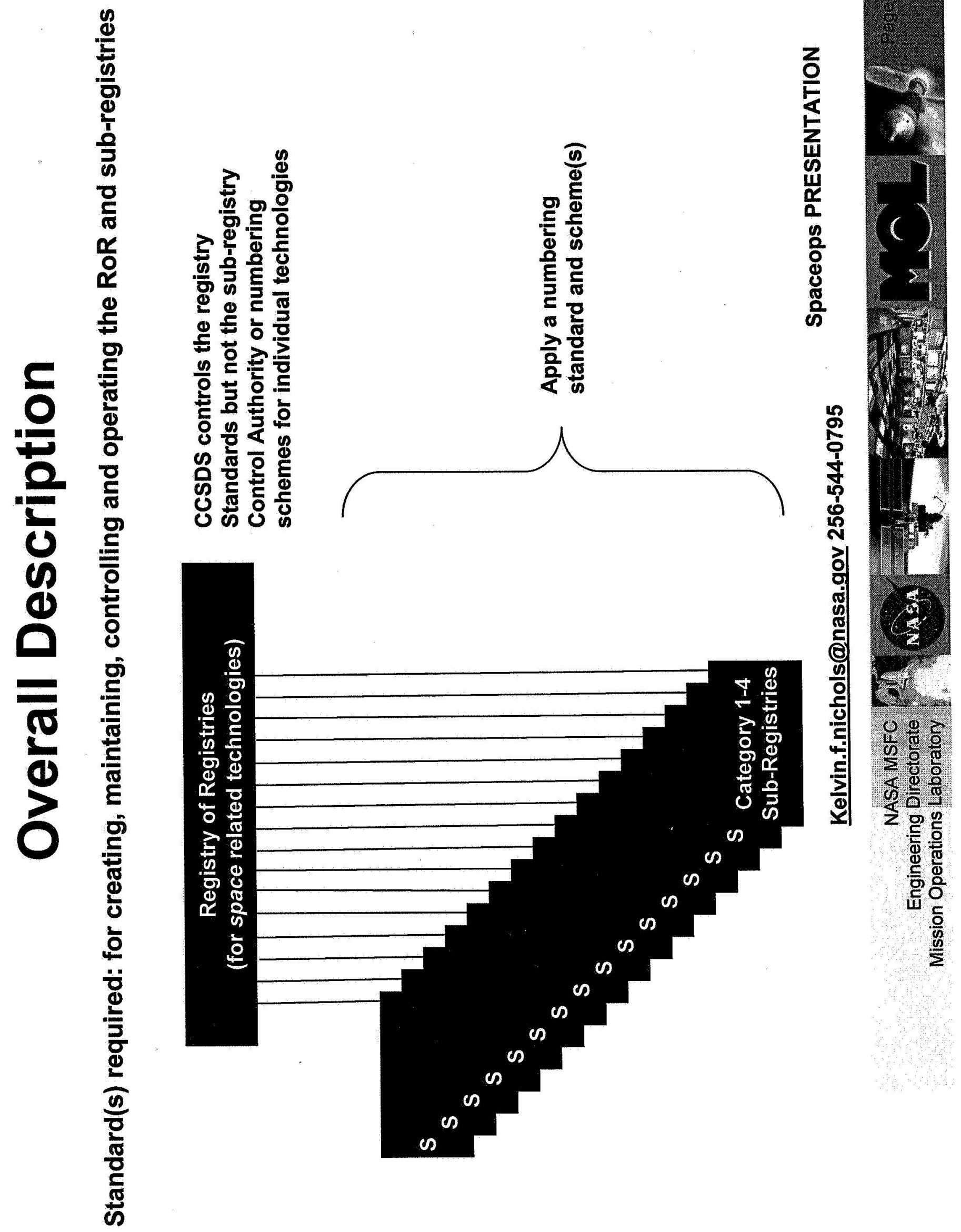




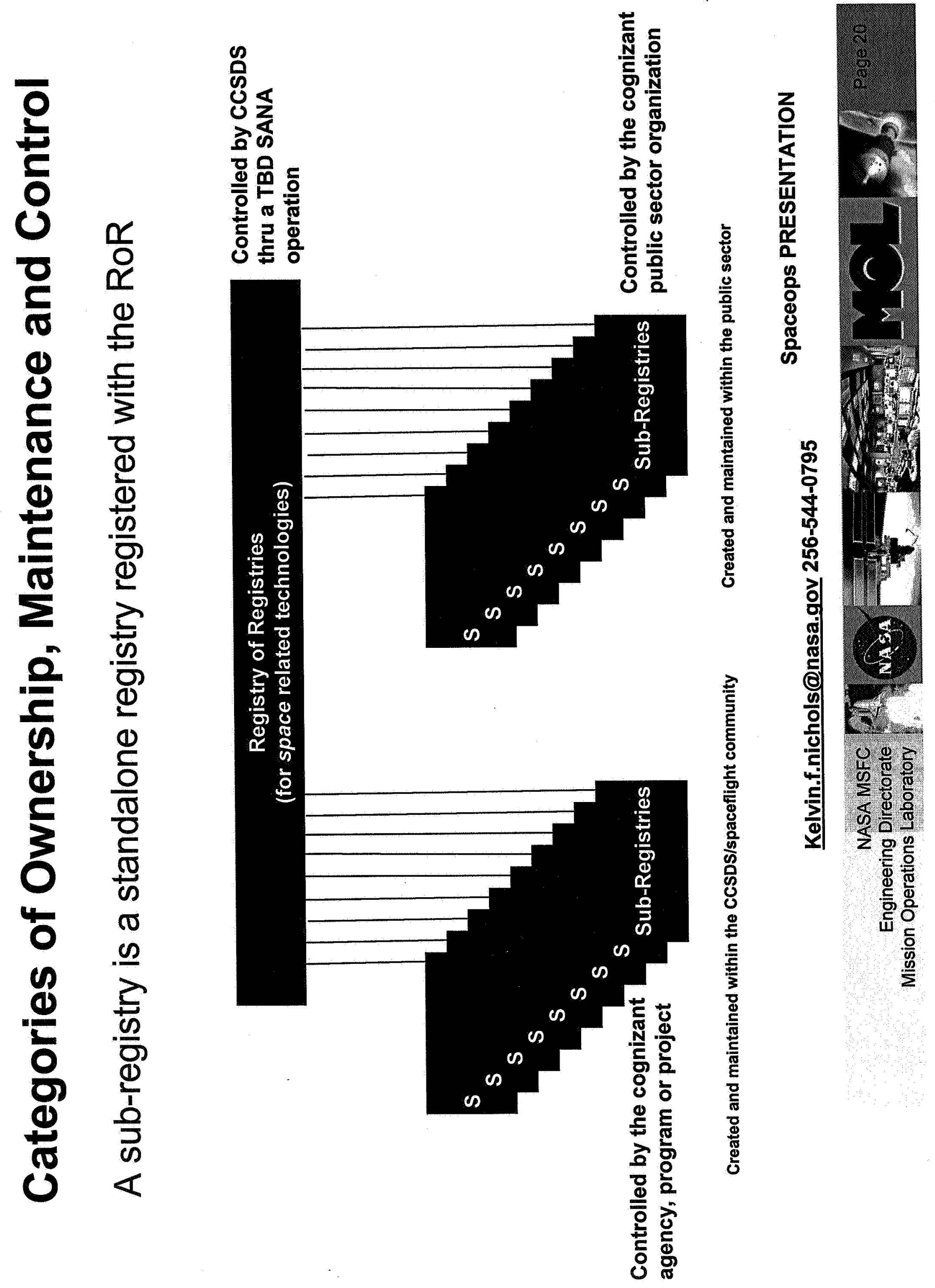




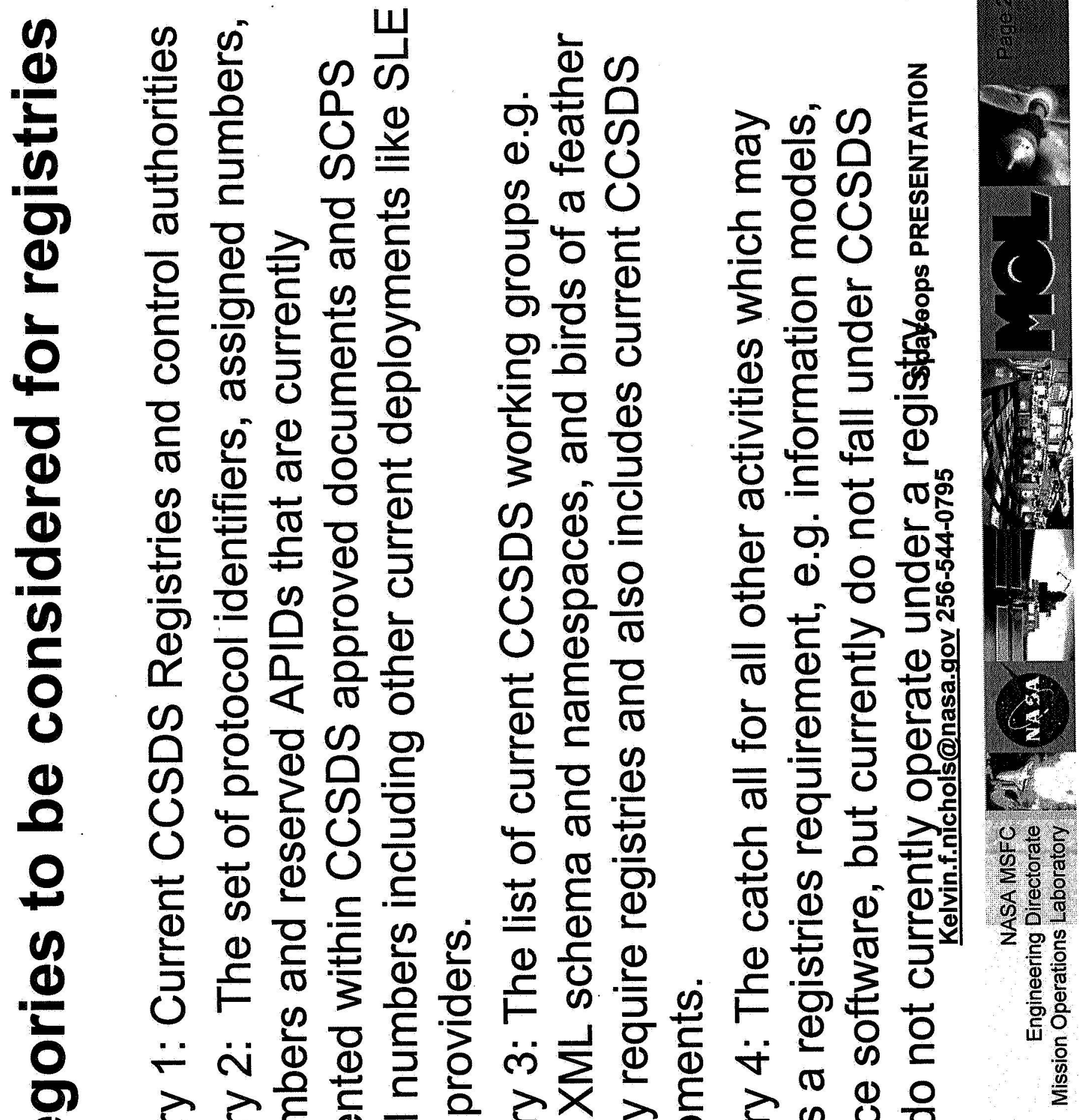

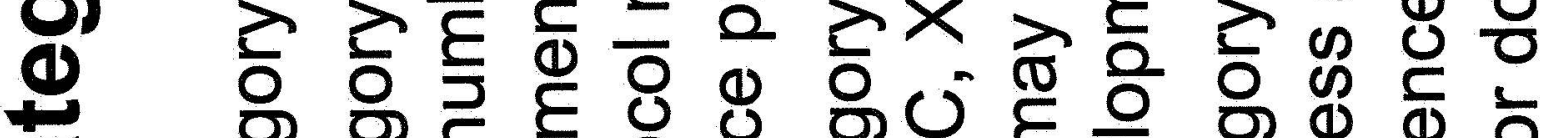

U

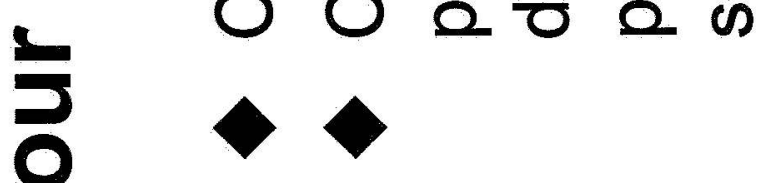

丩 


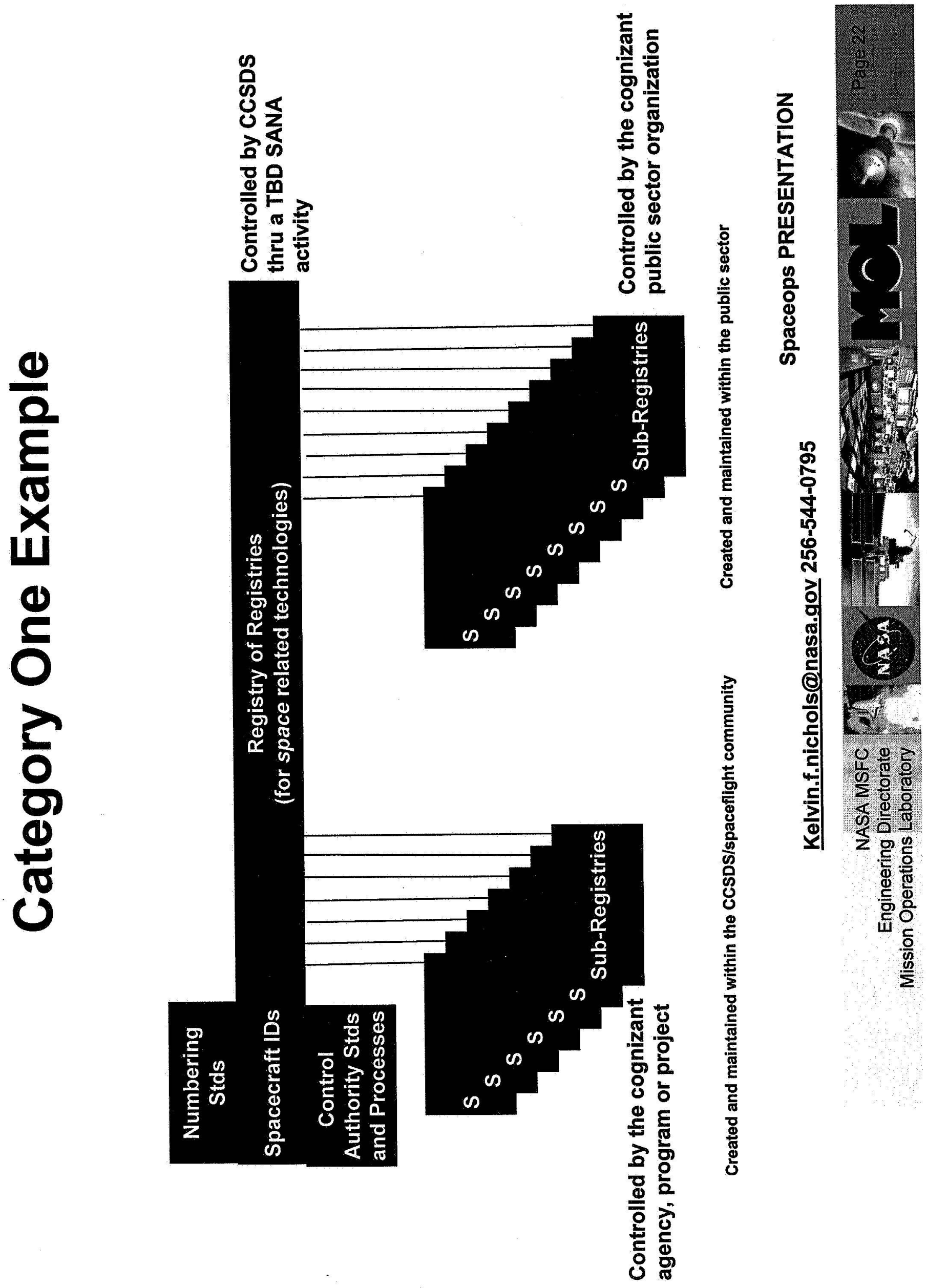



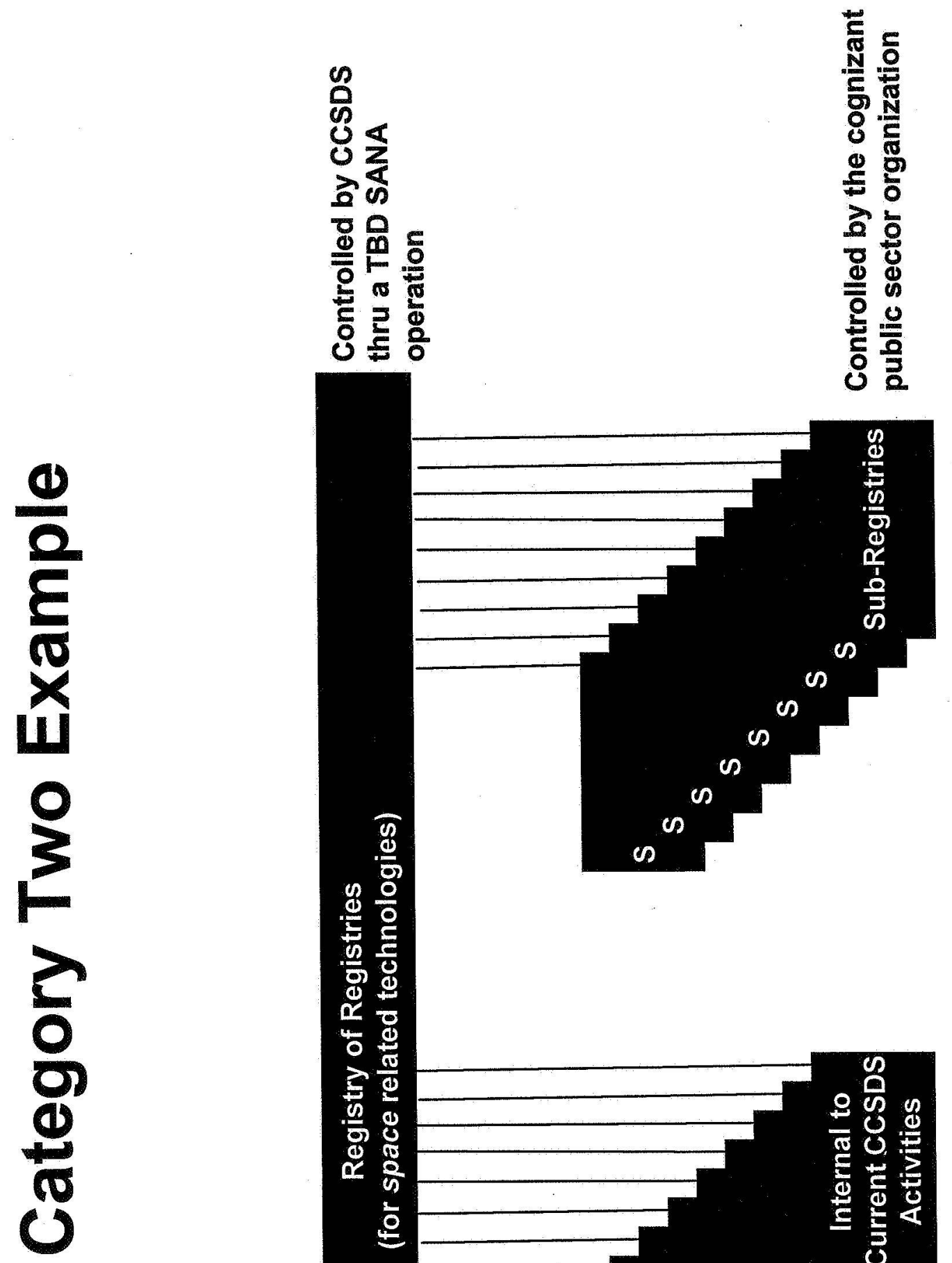

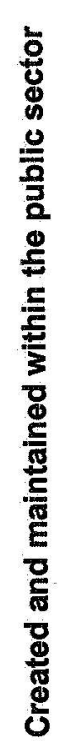
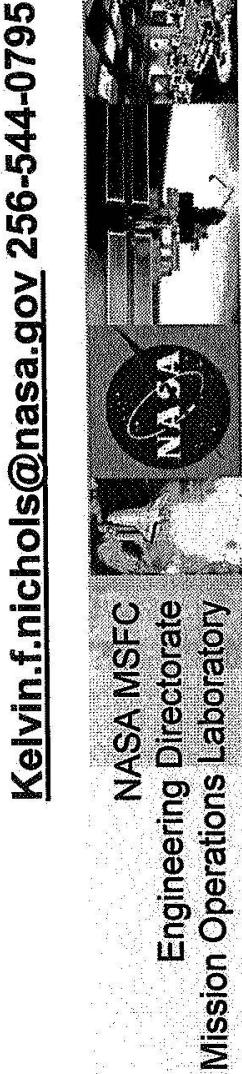

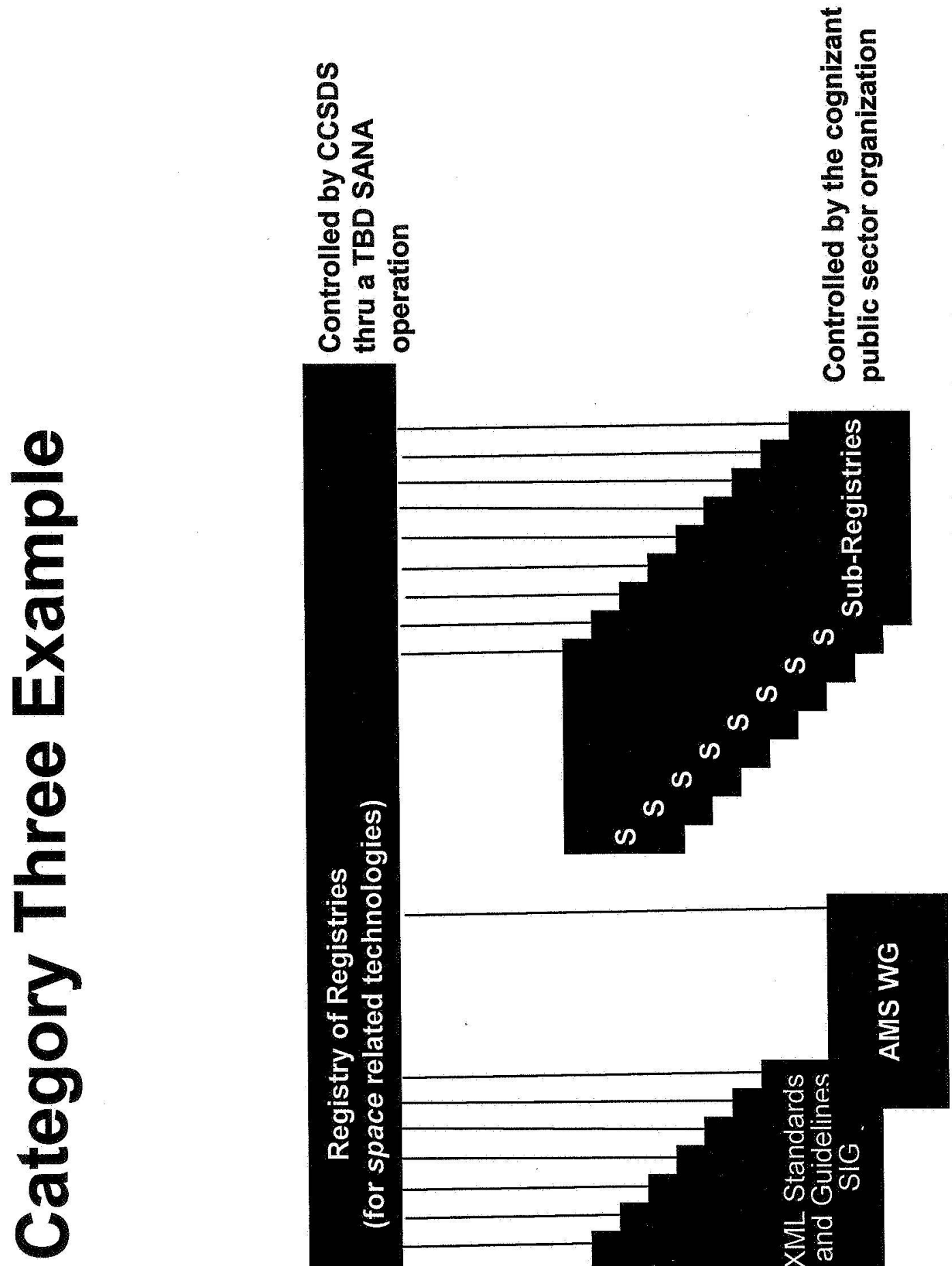


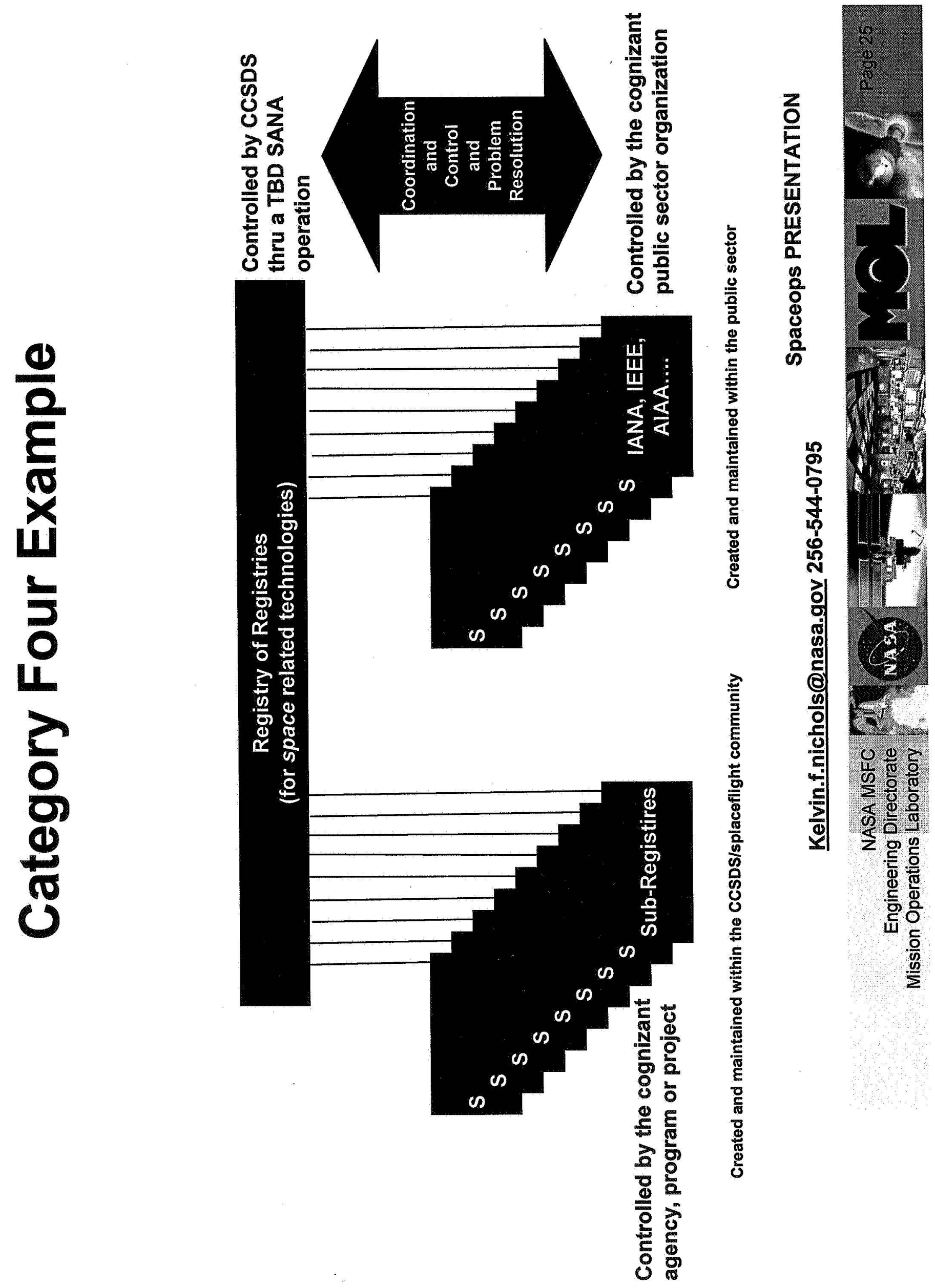




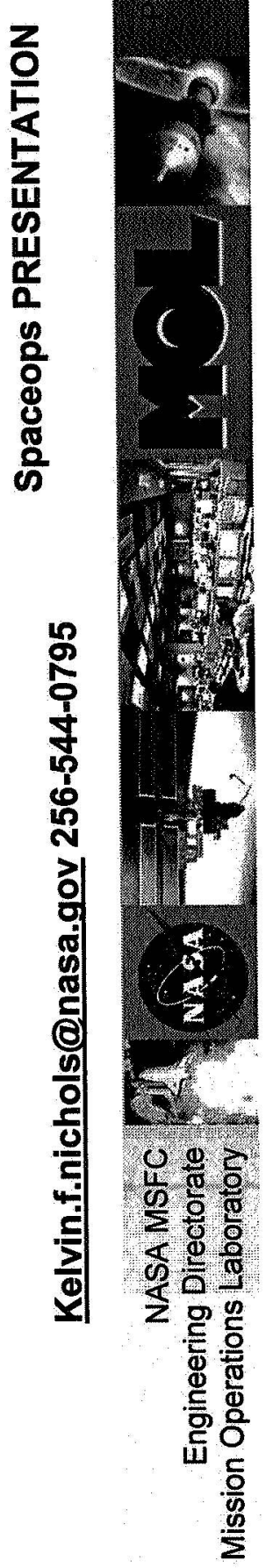




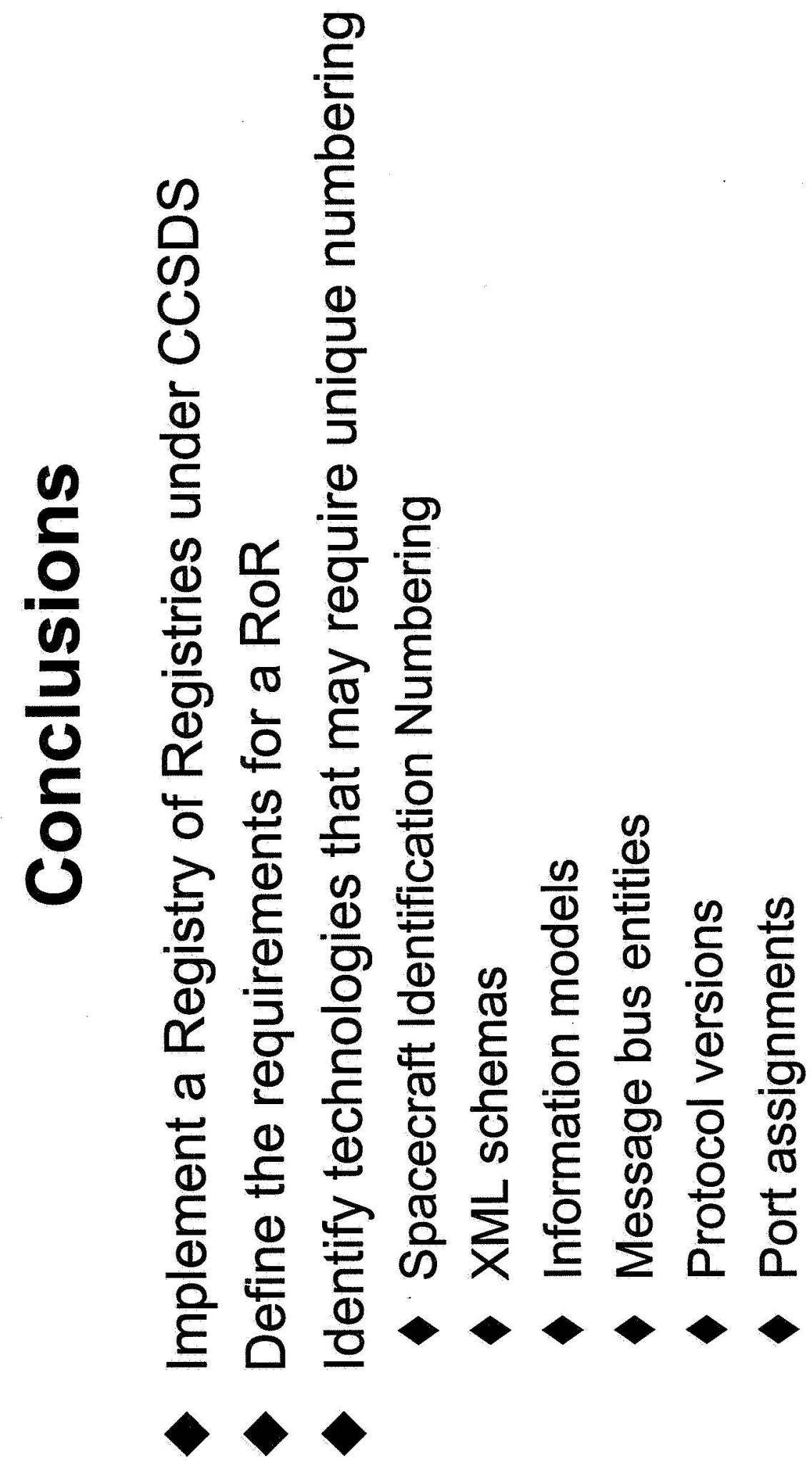




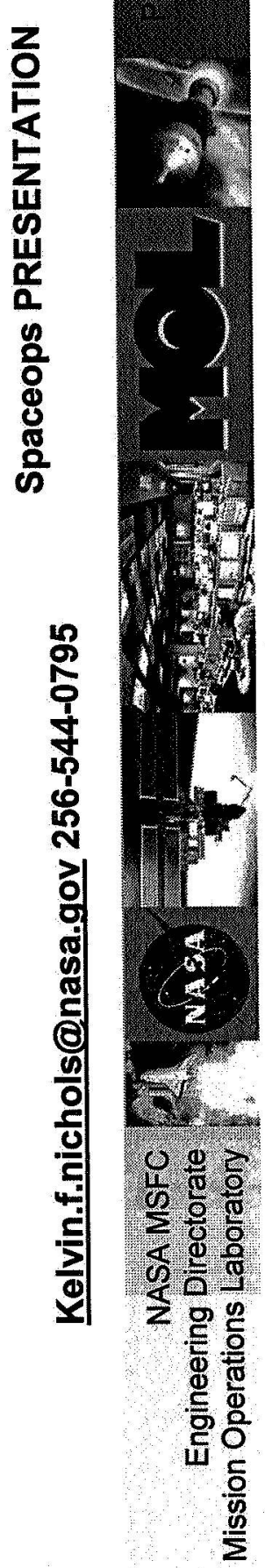

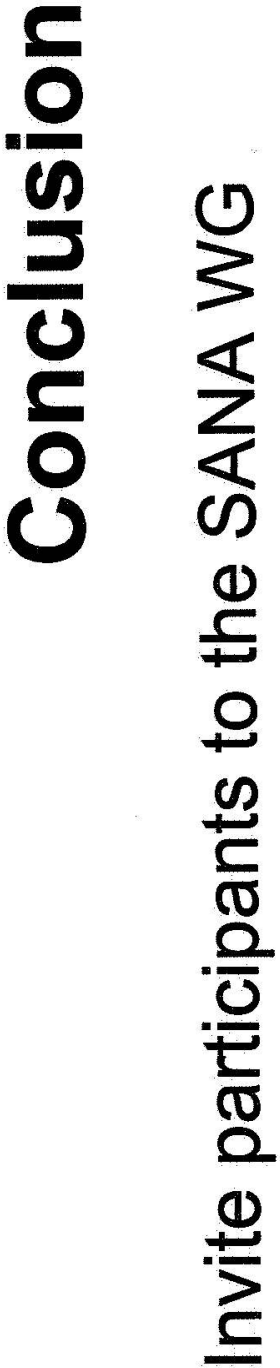

